\title{
Gyrokinetic calculations of diffusive and convective transport of $\alpha$ particles with a slowing down distribution function
}

\author{
C. Angioni and A.G. Peeters ${ }^{\dagger}$ \\ Max-Planck-Institut für Plasmaphysik, IPP-EURATOM Association, D-85748 Garching bei \\ München, Germany \\ ${ }^{\dagger}$ Center for Fusion, Space and Astrophysics, Physics department, University of Warwick, CV4 \\ 7AL, Coventry UK
}

(March 18, 2008)

Quasi-linear gyrokinetic calculations of the transport of fast $\alpha$ particles with a slowing down equilibrium distribution function in the trace limit are presented. Diffusive and convective contributions to the total flux are separated and their dependence on the ratio of the fast particle energy to the background plasma temperature is investigated. The results are compared with those obtained in the case an equivalent Maxwellian distribution function is assumed for the fast particles. On the basis of the gyrokinetic results, simple models for $\alpha$ particle transport are proposed for transport modelling purposes.

52.25.Fi, 52.30.Gz, 52.35.Qz, 52.55.Pi, 52.55.Fa

\section{INTRODUCTION}

The study of fast particle transport due to the background turbulence has received recently particular interest [1-6], as motivated by previous analytical and numerical works [7-13], as well as by the last stage of the ITER design [14], and related recent [15] and past [16-19] experimental results.

Here we investigate the transport of $\alpha$ particles, using a quasi-linear gyrokinetic model for core tokamak plasma turbulence in which, differently from previous works, the equilibrium distribution function of the fast particles is more realistically described by a slowing 
down distribution. The present study is dedicated to the transport of fast particles produced by usual core plasma microinstabilities, like ion temperature gradient (ITG) and trapped electron modes, which are mainly of electrostatic nature, and not to the transport produced by fast particle driven electromagnetic modes, like toroidal Alfvén eigenmodes (e.g. Refs. $[20,21])$. Here, we compute the transport produced by fluctuations of the electrostatic potential in the presence of an unperturbed magnetic confinement configuration of concentric flux surfaces. We shall not consider the important although different problem of the losses of fast particles generated by the presence of large scale magnetic perturbations, like magnetic islands, [22-24] or by the presence of magnetic perturbations leading to regions of stochasticity [25]. We mention that the latter problem is of interest not only in magnetic fusion plasmas but also in astrophysical plasmas (e.g. Ref. [26] and references therein).

Our work starts from the consideration that, as it will be shown, the concentration of $\alpha$ particles foreseen in the ITER standard scenario [27] is small enough that the passive tracer limit is practically applicable. Such a small concentration limit allows us to express the results in a physically meaningful form, separating the diffusive and the convective contributions to the particle flux in an unambiguous way. This is also particularly practical for transport modelling purposes. The numerical calculations presented here have been performed utilizing two gyrokinetic codes, GS2 (GKS) [28,29] and Gyrokinetics@Warwick (GKW) (LINART) [30,31]. These codes have been both appropriately modified for the purposes of the present work, by implementing a slowing down equilibrium distribution function, in addition to the usual Maxwellian distribution. The results obtained assuming a slowing down equilibrium distribution function are compared with those provided by an equivalent Maxwellian, namely by a Maxwellian whose temperature is such to provide the same second order moment $\left(v^{2}\right)$ as the slowing down distribution. The latter approach was already applied for the gyrokinetic description of $\alpha$ particle transport in a recent work [4].

We underline that the present work is dedicated to the study of $\alpha$ particle transport as fusion products, therefore of $\alpha$ particles largely more energetic than the background plasma. As we already mentioned, for these kind of particles, a slowing down distribution provides 
the most appropriate description. The present work is not dedicated specifically to the study of transport of He ash, although some of the present results can be applied to that purpose, as it will be shown. He ash transport is more appropriately described by a Maxwellian distribution at the same temperature of the background plasma, namely, He ash can be treated more appropriately in the same way as a usual plasma impurity.

In the next Section, the gyrokinetic equation with a slowing down equilibrium distribution function is derived, and is compared to that with a Maxwellian equilibrium distribution. The consequences of the new terms in this gyrokinetic equation on the expression of the particle flux are identified analytically in Sec. III, by introducing a kernel function which captures the dependence of diffusivity on the energy variable, independently of the specific choice of the unperturbed distribution function. In Sec. IV the reference case is defined and the results of a scan in the $\alpha$ particle concentration are presented. In Sec. V, the dependence of the diffusivity on the energy variable in the velocity space is investigated. In Sec. VI and Sec. VII, the numerical results related to the dependence of the diffusion and convective coefficients as a function the background plasma temperature are presented. In Sec. VIII, a method which utilizes a fitting formula of the energy dependence of the kernel function for the calculation of the transport coefficients for any choice of the unperturbed distribution function is outlined and demonstrated. In Sec. IX, a transport model for $\alpha$ particles summarizing the code results is derived and compared with an analogous model for He ash. In Sec. X, the comparison of present results with previous results published in the literature is discussed, and, finally, in Sec. XI, the conclusions of the present work are drawn.

\section{THE GYROKINETIC EQUATION FOR AN EQUILIBRIUM SLOWING DOWN DISTRIBUTION FUNCTION}

The gyrokinetic equation usually considered and solved in numerical codes assumes an equilibrium (unperturbed) Maxwellian distribution. While such an equilibrium distribution 
is appropriate for thermal particle species, it does not describe appropriately fast particles in the slowing down process. More specifically, for fusion $\alpha$ particles, a realistic equilibrium distribution is an isotropic slowing down distribution function, namely a slowing down distribution dependent only on the particle kinetic energy and independent of the velocity pitch angle. This is provided by the solution of the Fokker-Planck equation with an isotropic particle source at a single energy, as a $\mathrm{D}-\mathrm{T}$ fusion reaction is for $\alpha$ particles. The analytic form of such a distribution function has been derived and presented in the literature [32-34]. Such a distribution can also be considered for beam ions which do not have a strong anisotropy in the velocity pitch angle [35]. We start our derivation here from the linearized gyrokinetic equation in a form in which no assumption is made yet on the actual energy dependence of the unperturbed distribution function, while assuming that it is independent of the velocity pitch angle, or the magnetic moment (e.g. [36,37]). We note that the extension of the present derivation to a nonlinear $\delta f$ equation is straightforward, since the nonlinear terms do not require any modification. From Eq. (35) of Ref. [37], dropping terms involving the derivative of the unperturbed distribution function versus the magnetic moment, the linearized gyrokinetic equation reads

$$
\frac{\partial h_{\alpha}}{\partial t}+\left(v_{\|} \mathbf{e}_{z}+\mathbf{v}_{d} \cdot\right) \nabla h_{\alpha}=-e_{\alpha} \frac{\partial F_{0 \alpha}}{\partial E} \frac{\partial\langle U\rangle}{\partial t}+\frac{c}{B}\left\langle\nabla U \times \mathbf{e}_{z}\right\rangle \cdot \nabla F_{0 \alpha}
$$

where $v_{\|}$is the velocity parallel to the magnetic field line, $\mathbf{v}_{d}$ is the gyrocenter perpendicular velocity, combination of the $\nabla B$ and curvature drifts, \langle\rangle is the gyroaverage operator, and $U$ is the generalized potential

$$
U=\phi-\frac{1}{c} \mathbf{v} \cdot \mathbf{A}
$$

The distribution function $h_{\alpha}$ is related to the perturbed distribution function $f_{\alpha}$ by the relation

$$
h_{\alpha}=f_{\alpha}-e_{\alpha} \frac{\partial F_{0 \alpha}}{\partial E} \phi+\frac{1}{B} \mathbf{A} \times \mathbf{e}_{z} \cdot \nabla F_{0 \alpha} .
$$

Here $F_{0 \alpha}$ and $f_{\alpha}$ are respectively the equilibrium (unperturbed) and the perturbed distribution functions of the generic species with index $\alpha$, which can be identified with the fusion $\alpha$ 
particles. We have defined a local set of right-handed perpendicular axes, $\mathbf{e}_{x}, \mathbf{e}_{y}, \mathbf{e}_{z}$, where $\mathbf{e}_{z}=\mathbf{B} / B$ is aligned with the magnetic field line, $\mathbf{e}_{y}$ is locally perpendicular to the field line and belongs to the magnetic flux surface, and $\mathbf{e}_{x}$ is in the radial direction. The energy variable is defined as $E=m_{\alpha} v^{2} / 2$, where $m_{\alpha}$ and $e_{\alpha}$ in previous equations are respectively the mass and the charge of the species $\alpha$. Finally, $\phi$ and $\mathbf{A}$ are the perturbed electrostatic and magnetic vector potentials.

Assuming that the equilibrium distribution function is a Maxwellian,

$$
F_{M \alpha}=\frac{n_{\alpha}}{\pi^{3 / 2} v_{t h \alpha}^{3}} e^{-v^{2} / v_{t h \alpha}^{2}}
$$

the derivatives of $F_{0 \alpha}$ occurring in Eq. (1) take the well known forms

$$
\frac{d F_{M \alpha}}{d E}=-\frac{F_{M \alpha}}{T_{\alpha}}
$$

and

$$
\frac{d F_{M \alpha}}{d r}=F_{M \alpha}\left[\frac{d \log n_{\alpha}}{d r}+\left(\frac{E}{T_{\alpha}}-\frac{3}{2}\right) \frac{d \log T_{\alpha}}{d r}\right]
$$

where $v_{t h \alpha}^{2}=2 T_{\alpha} / m_{\alpha}$, with $T_{\alpha}$ the temperature of the species $\alpha$.

In the presence of a background distribution function which is not Maxwellian, the expressions for $d F_{0 \alpha} / d E$ and $d F_{0 \alpha} / d r$ have to be recomputed and the final form of the gyrokinetic equation modified consistently.

We consider an equilibrium distribution function as given by a slowing down distribution function $F_{0 \alpha}=F_{S}$, with

$$
F_{S}=\frac{n_{\alpha}}{4 \pi I_{2}} \frac{H\left(v_{\alpha}-v\right)}{v_{c}^{3}+v^{3}}
$$

Here $v_{\alpha}$ is the maximum fast particle velocity, namely the birth velocity of $\alpha$ particles, corresponding to an energy $E_{\alpha}=m_{\alpha} v_{\alpha}^{2} / 2=3.5 \mathrm{MeV}$ for fusion $\alpha$ particles, and $v_{c}$ is the slowing-down critical velocity (we do not quote here the index $\alpha$ to lighten the symbols). This is related to the background plasma electron thermal velocity $v_{\text {the }}$ by the relation

$$
v_{c}^{3}=\frac{3 \sqrt{\pi}}{4} \frac{m_{e}}{m_{\alpha}} Z_{I} v_{t h e}^{3}
$$


where $Z_{I}$ is an effective charge where charges are weighted inversely over the corresponding ion mass, $Z_{I}=m_{\alpha} \sum_{i} n_{i}\left(Z_{i}^{2} / m_{i}\right) / n_{e}$, and equal to $5 / 3$ for a mixture of $50 \% \mathrm{D}$ and $50 \% \mathrm{~T}$. As customary, in Eq. (7), the quantity $I_{2}=I_{2}\left(v_{c} / v_{\alpha}\right)$ is introduced in order to ensure that the integral in the velocity space of the distribution gives the actual zero-order moment $n_{\alpha}$, namely the density (we refer to Sec. II of the recent Ref. [4] for a more complete derivation),

$$
I_{2}\left(\frac{v_{c}}{v_{\alpha}}\right)=\frac{1}{3} \log \left(\frac{v_{\alpha}^{3}}{v_{c}^{3}}+1\right) .
$$

The expression of the slowing down distribution function presented in Eq. (7) is appropriate for large ratios of the birth energy of the energetic particles to the background plasma temperature, namely $E_{\alpha} / T_{e}>>1$. This ratio is larger than 100 in the range of temperatures foreseen in a fusion reactor plasma, which fully justifies the expression in Eq. (7) for the $\alpha$ particle distribution function. In order to fully explore the dependence on energy of the transport coefficients, here the same expression of the distribution function is used also in the limit of $T_{e} \sim T_{\alpha}$, with no aim of consistency with the actual form of the distribution function in those unrealistic conditions.

The derivative versus energy of such a distribution, excluding the discontinuity at the upper energy boundary, reads

$$
\frac{d \log \left(F_{S}\right)}{d \hat{E}}=-\frac{3}{2} \frac{\hat{E}^{0.5}}{\hat{E}^{1.5}+\hat{E}_{c}^{1.5}}, \text { for } 0 \leq \hat{E}<1,
$$

where $E_{c}=m_{\alpha} v_{c}^{2} / 2$, and where we have introduced the dimensionless energy variable $\hat{E}=$ $E / E_{\alpha}$, and consistently $\hat{E}_{c}=E_{c} / E_{\alpha}$. The radial derivative reads,

$$
\frac{d \log \left(F_{S}\right)}{d r}=\frac{d \log n_{\alpha}}{d r}+\mathcal{K}_{S}(\hat{E}) \frac{d \log \left(\hat{E}_{c}\right)}{d r}
$$

where we have introduced the function

$$
\mathcal{K}_{S}(\hat{E})=\frac{3}{2}\left[\frac{1}{\log \left(1 / \hat{E}_{c}^{1.5}+1\right)\left(1+\hat{E}_{c}^{1.5}\right)}-\frac{\hat{E}_{c}^{1.5}}{\hat{E}_{c}^{1.5}+\hat{E}^{1.5}}\right] .
$$

At this point, it is practical to introduce also the function 


$$
\mathcal{H}_{S}(\hat{E})=-\frac{d \log \left(F_{S}\right)}{d \hat{E}}=\frac{3}{2} \frac{\hat{E}^{0.5}}{\hat{E}^{1.5}+\hat{E}_{c}^{1.5}} .
$$

We note that in the case of an equivalent Maxwellian, namely a Maxwellian whose temperature $T_{\alpha}$ is such that

$$
\int F_{M} v^{2} \mathrm{~d}^{3} \mathbf{v}=\int F_{s} v^{2} \mathrm{~d}^{3} \mathbf{v}
$$

the corresponding expressions for the functions $\mathcal{H}_{M}$ and $\mathcal{K}_{M}$ read

$$
\mathcal{H}_{M}=-\frac{d \log \left(F_{M}\right)}{d \hat{E}}=1
$$

and

$$
\mathcal{K}_{M}=\left(\hat{E}-\frac{3}{2}\right) \frac{d \log T_{\alpha}}{d \log E_{c}}
$$

where we define the dimensionless energy variable for the Maxwellian in the customary form $\hat{E}=E / T_{\alpha}$. We recall that an analytical expression for the equivalent temperature $T_{\alpha}$ can be derived (see e.g. Eq. (15) in Ref. [4]). The radial derivative of the equivalent Maxwellian can be expressed in a form absolutely analogous to that of Eq. (11),

$$
\frac{d \log F_{M \alpha}}{d r}=\frac{d \log n_{\alpha}}{d r}+\mathcal{K}_{M} \frac{d \log E_{c}}{d r} .
$$

We underline that in the previous expression, it has been taken into account that the equivalent Maxwellian temperature $T_{\alpha}$ is a function of the slowing down critical energy $E_{c}$ through the relationship provided by Eq. (14).

In the present work, we limit the investigation to the fast particle transport produced by electrostatic microinstabilities in a simple circular $s-\alpha$ geometry. In this limit, for a single toroidal mode in the ballooning representation for an $s-\alpha$ equilibrium [38], Eq. (1) can be written in the following form, analogous to Eq. (2) of Ref. [28],

$$
\frac{\partial \hat{g}_{\alpha}}{\partial t}+\frac{v_{\|}}{q R} \frac{\partial \hat{g}_{\alpha}}{\partial \theta}+i \omega_{d \alpha} \hat{g}_{\alpha}=\frac{e_{\alpha}}{E_{\alpha}} F_{S \alpha} J_{0}\left[\mathcal{H}_{S}(\hat{E}) \frac{\partial \hat{\phi}(\theta)}{\partial t}+i \omega_{S *} \hat{\phi}(\theta)\right],
$$

where $q$ is the safety factor, $R$ is the major radius, and $\theta$ is the extended ballooning angle and $J_{0}=J_{0}\left(k_{\perp} v_{\perp} / \Omega_{\alpha}\right)$. The non-adiabatic part of the perturbed distribution function $\hat{g}_{\alpha}$ is related to the perturbed distribution function $\hat{f}_{\alpha}$ by 


$$
\hat{g}_{\alpha}=\hat{f}_{\alpha}+e_{\alpha} F_{S \alpha} \mathcal{H}_{S}(\hat{E}) \hat{\phi}(\theta) .
$$

In Eq. (18) we have introduced a slowing down diamagnetic frequency

$$
\omega_{S *}=\frac{k_{\theta} E_{\alpha}}{e_{\alpha} B R}\left[\frac{R}{L_{n \alpha}}+\mathcal{K}_{S}(\hat{E}) \frac{R}{L_{E c}}\right]
$$

In the above expression, we have introduced the customary dimensionless logarithmic gradients $R / L_{n \alpha}=-R d \log n_{\alpha} / d r$ and $R / L_{E c}=-R d \log E_{c} / d r$. We note that, provided that the ion species concentrations are homogeneous along the minor radius, namely $Z_{I}$ is constant, $R / L_{E c}=R / L_{T e}$, as it can be easily deduced from Eq. (8). The gyrokinetic equation Eq. (18) has absolutely analogous form to the usual gyrokinetic equation with an equilibrium Maxwellian distribution (e.g., see specifically Eq. (2) in Ref. [28] for comparison). The gyrokinetic equation with an equilibrium Maxwellian distribution is indeed directly obtained with the obvious replacement of $E_{\alpha}$ by $T_{\alpha}$ in the right hand sides of Eqs. (18) and (20), and using consistently $\mathcal{H}_{M}$ and $\mathcal{K}_{M}$, defined by Eqs. (15) and (16), at the place of $\mathcal{H}_{S}(\hat{E})$ and $\mathcal{K}_{S}(\hat{E})$ respectively.

We have modified the GS2 code, as well as the GKW code, in order to solve the above mentioned equation (18). In the case of the equilibrium slowing down distribution function, we have considered an energy grid from 0 up to $E_{\alpha}$ which excludes the upper boundary $E=E_{\alpha}$, namely $0 \leq \hat{E}<1$, and by this neglects the discontinuity at $E=E_{\alpha}$ in the energy derivative. We note that the two codes utilized in these work make a different choice of variables for the discretization of the velocity space. While GS2 discretizes the variables $\hat{E}$ and $\lambda=\mu B_{0} / E$, with $\mu=m_{\alpha} v_{\perp}^{2} /(2 B)$, the GKW code discretizes the variables $v_{\|}$and $\mu$. Also the numerical schemes are different. More importantly, however, the implementation was made independently by the authors, such that the benchmark gives strong support to the correctness of the results. 


\section{PARTICLE FLUX FOR A SLOWING DOWN FAST PARTICLE}

Here we investigate analytically the role played by the new terms in the gyrokinetic equation on the expression of the particle flux of the $\alpha$ particles. We shall make the assumption that the fast particle concentration is small enough, that its contribution to the Poisson equation can be considered negligible (passive tracer limit). Introducing the complex eigenfrequency $\omega$, in such a way that $\partial / \partial t \rightarrow-i \omega$, and developing the right hand side of the gyrokinetic equation Eq. (18), including Eq. (20), we obtain

$$
-i \omega \hat{g}_{\alpha}+\frac{v_{\|}}{q R} \frac{\partial \hat{g}_{\alpha}}{\partial \theta}+i \omega_{d \alpha} \hat{g}_{\alpha}=i \frac{e_{\alpha} \omega_{D \alpha}}{E_{\alpha}} F_{\alpha} J_{0} \hat{\phi}\left[\frac{R}{L_{n \alpha}}+\mathcal{K}_{S}(\hat{E}) \frac{R}{L_{E c}}-\frac{\omega}{\omega_{D \alpha}} \mathcal{H}_{S}(\hat{E})\right],
$$

where we have introduced the frequency $\omega_{D \alpha}=\left(k_{\theta} E_{\alpha}\right) /\left(e_{\alpha} B R\right)$ for normalization purposes.

We observe that the solution $\hat{g}_{\alpha}$ of the equation can be expressed in the form,

$$
\hat{g}_{\alpha}=\frac{e_{\alpha}}{E_{\alpha}} F_{\alpha} h_{D}\left[\frac{R}{L_{n \alpha}}+\mathcal{K}_{S}(\hat{E}) \frac{R}{L_{E c}}-\frac{\omega}{\omega_{D \alpha}} \mathcal{H}_{S}(\hat{E})\right]
$$

where the function $h_{D}$ is the solution of the following equation in $\theta$,

$$
\frac{v_{\|}}{q R} \frac{\partial h_{D}}{\partial \theta}+i\left(\omega_{d \alpha}-\omega\right) h_{D}=i \omega_{D \alpha} J_{0} \hat{\phi}(\theta)
$$

For particle species in small enough concentration to be negligible in the Poisson equation, this procedure is completely general, and the function $h_{D}$ is completely independent of the actual shape of the equilibrium distribution function.

This equation can be solved symbolically in the form

$$
h_{D}=\frac{-\omega_{D \alpha}}{\omega-k_{\| \mid} v_{\|}-\omega_{d \alpha}} J_{0} \hat{\phi}
$$

where we have introduced a parallel wave number $k_{||} \hat{g}=\partial \hat{g} / \partial \theta /(q R)$, and treated it formally as a fixed number. Obviously the real solution involves the one dimensional integration along the field line, and this is performed consistently in the codes. However, as we shall also discuss later, the symbolic solution in Eq. (21) is useful to identify the resonance condition to be fulfilled for $h_{D}$ to be large, namely that $\omega_{d \alpha}+k_{\|} v_{\|}$of the fast particles be comparable to the eigenvalue $\omega$ of the background plasma instability. 
The non-adiabatic part of the density perturbation $\tilde{n}_{n . a}$ is provided by the integral of $\hat{g}$ in the velocity space. This reads

$$
\begin{aligned}
& \tilde{n}_{n . a .}=\frac{e_{\alpha}}{E_{\alpha}} . \\
& {\left[\left(\int h_{D} F_{\alpha} J_{0} d^{3} \mathbf{v}\right) \frac{R}{L_{n \alpha}}+\left(\int h_{D} F_{\alpha} \mathcal{K}_{S}(\hat{E}) J_{0} d^{3} \mathbf{v}\right) \frac{R}{L_{E c}}-\left(\int h_{D} F_{\alpha} \mathcal{H}_{S}(\hat{E}) J_{0} d^{3} \mathbf{v}\right) \frac{\omega}{\omega_{D \alpha}}\right]}
\end{aligned}
$$

The quasi-linear particle flux produced by the fluctuating $E \times B$ velocity at a given wave number $k_{\theta}$ is given by $\Gamma_{k}=\left\langle\operatorname{Im}\left(k_{\theta} \tilde{\phi}^{\dagger} \tilde{n} / B\right)\right\rangle$, where the symbol $\dagger$ indicates the complex conjugate, and the brackets \langle\rangle indicate the flux surface average.

By replacing the expression for $\tilde{n}$ presented above, the quasi-linear particle flux for a given wave number $k_{\theta}$ can be written in the form

$$
\frac{R \Gamma_{k}}{n_{\alpha}}=D_{k} \frac{R}{L_{n \alpha}}+D_{E k} \frac{R}{L_{E c}}+R V_{p k}
$$

where

$$
\begin{gathered}
D_{k}=\left\langle\frac{k_{\theta} R}{n_{\alpha} B} \frac{e_{\alpha}}{E_{\alpha}} \operatorname{Im}\left\{\int \mathrm{d}^{3} \mathbf{v} J_{0} F_{\alpha} h_{D} \hat{\phi}^{\dagger}\right\}\right\rangle \\
D_{E k}=\left\langle\frac{k_{\theta} R}{n_{\alpha} B} \frac{e_{\alpha}}{E_{\alpha}} \operatorname{Im}\left\{\int \mathrm{d}^{3} \mathbf{v} J_{0} F_{\alpha} \mathcal{K}(\hat{E}) h_{D} \hat{\phi}^{\dagger}\right\}\right\rangle
\end{gathered}
$$

and

$$
R V_{p k}=-\left\langle\frac{k_{\theta} R}{n_{\alpha} B} \frac{e_{\alpha}}{E_{\alpha}} \frac{\omega}{\omega_{D \alpha}} \operatorname{Im}\left\{\int \mathrm{d}^{3} \mathbf{v} J_{0} F_{\alpha} \mathcal{H}(\hat{E}) h_{D} \hat{\phi}^{\dagger}\right\}\right\rangle
$$

Provided that the unperturbed distribution function depends only on the energy variable, by noting that the order of integration on the energy variable and on the ballooning angle $\theta$ in the flux surface average can be inverted, and by introducing the following integral of the function $h_{D}$, over the customary pitch angle variable $\lambda=\mu / E$ (or other analogous velocity variable),

$$
G_{D k}=\left\langle\frac{k_{\theta} R}{n_{\alpha} B} \frac{e_{\alpha} \omega_{D \alpha}}{E_{\alpha}} \operatorname{Im}\left\{\int \frac{B d \lambda}{\sqrt{2(1-\lambda B)}} J_{0} h_{D} \hat{\phi}^{\dagger}\right\}\right\rangle
$$


we obtain that the diffusion and convective coefficients can be expressed in the compact forms

$$
\begin{gathered}
D_{k}=\frac{1}{n_{\alpha}} \int G_{D k}(E) F_{\alpha}(2 \pi \sqrt{E}) d E \\
D_{E k}=\frac{1}{n_{\alpha}} \int G_{D k}(E) F_{\alpha} \mathcal{K}(\hat{E})(2 \pi \sqrt{E}) d E \\
R V_{p k}=-\frac{1}{n_{\alpha}} \frac{\omega}{\omega_{D \alpha}} \int G_{D k}(E) F_{\alpha} \mathcal{H}(\hat{E})(2 \pi \sqrt{E}) d E .
\end{gathered}
$$

We remind that the unperturbed distribution function is such that $\int F_{\alpha}(2 \pi \sqrt{E}) d E=n_{\alpha}$.

Finally, it is useful to define the following coefficients

$$
C_{E k}=\frac{D_{E k}}{D_{k}}, \quad C_{p k}=\frac{R V_{p k}}{D_{k}}
$$

in such a way that the quasi-linear particle flux can be expressed in the form

$$
\frac{R \Gamma_{k}}{n_{\alpha}}=D_{k}\left(\frac{R}{L_{n \alpha}}+C_{E k} \frac{R}{L_{T e}}+C_{p k}\right),
$$

where we have considered that $R / L_{E c}=R / L_{T e}$ for homogeneous charge concentrations of the thermal ion species, as given by the dependence of the critical energy $E_{c}$ on the electron temperature defined in Eq. (8).

From this derivation, we find that the $\alpha$ particle flux is made of three contributions, one perfectly diffusive, proportional to its logarithmic density gradient, and two convective terms, one proportional to the electron temperature logarithmic gradient, and one which is a pure convective contribution. We shall call $C_{E k}$ the thermal convection coefficient, and $C_{p k}$ the pure convection coefficient. The auxiliary functions $\mathcal{K}(\hat{E})$ and $\mathcal{H}(\hat{E})$, introduced in the previous Section and defined respectively through the radial and the energy derivatives of the equilibrium distribution function $F_{\alpha}$, like in Eq. (11) and in Eq. (13), occur directly in the expressions of the thermal convection, Eq. (28) for $D_{E k}$, and of the pure convection, Eq. (29) for $R V_{p k}$.

It is interesting to note that, with respect to the case of a Maxwellian distribution, with a slowing down distribution the so-called thermodiffusive contribution to the particle 
flux, namely that proportional to the logarithmic gradient of the temperature of the same particle species, is replaced by a term proportional to the logarithmic gradient of the electron temperature. This justifies why we prefer to refer to this term as thermal convection rather than thermodiffusion.

We observe also that the kernel function $G_{D k}$, defined as the flux surface average of the integral over the pitch angle variable $\lambda$ of the function $h_{D}$ in Eq. (26), is completely independent of the specific choice of the equilibrium distribution function $F_{\alpha}$. Therefore it can be considered as a Green function. Once the energy dependence of such a function is known, all the diffusive and convective transport coefficients, for whatever kind of choice of the equilibrium distribution function $F_{\alpha}$, can be computed as convolutions over the energy variable of this function with the equilibrium distribution and with the related auxiliary functions $\mathcal{K}(\hat{E})$ and $\mathcal{H}(\hat{E})$.

In the following sections we compute the quasi-linear particle flux, as well as the kernel function $G_{D}$, with the modified versions of GS2 and GKW. We investigate the behavior of the coefficients $D, C_{E}$ and $C_{p}$ as a function of the electron temperature $T_{e}$, while keeping fixed $E_{\alpha}=3500 \mathrm{keV}$. We note that equivalent plots can be produced in terms of the parameter $E_{\alpha} / T_{e}$. The method applied to separate diffusive and convective contributions to the total particle flux is the same already applied for gyrokinetic studies of impurity transport $[39,40]$.

We describe $\alpha$ particles with a slowing down distribution function in negligible charge concentration (passive limit), as well as, for comparison, with an equivalent Maxwellian distribution, namely a Maxwellian distribution with an equivalent temperature $T_{\alpha}$, defined by Eq. (14). Table 1 shows the values of $T_{e}, E_{\alpha} / T_{e}, T_{\alpha}$ and $T_{\alpha} / T_{e}$ for the set of values of $T_{e}$ which has been used for the numerical calculations with the GS2 code.

\section{SCAN ON THE $\alpha$ PARTICLE CONCENTRATION}

The starting point of our work has been to establish a relevant reference case for the background plasma. We have chosen the following parameters $r / a=0.5, R / a=3, q=1.4$, 
$s=0.8, T_{e}=T_{i}, R / L_{T i}=R / L_{T e}=6, R / L_{n}=3$, electrostatic, collisionless at $k_{\theta} \rho_{i}=0.18$. In the previous expressions, $r$ is the minor radius, $a$ is the corresponding minor radius of the last close flux surface, $q$ is the safety factor and $s$ is the magnetic shear, while $\rho_{i}=c_{i} / \Omega_{i}$, where $c_{i}=\left(T_{i} / m_{i}\right)^{0.5}$ and $\Omega_{i}=e_{i} B / m_{i}$ is the cyclotron frequency. These physical parameters have the following properties. First they are average values of the parameters which are measured at mid-radius in low collisionality plasmas in a large tokamak like ASDEX Upgrade, second they are approximately the values (rounded to the closest integer) obtained by GLF23 [41] at mid-radius in a ITER standard scenario simulation [42], third they fulfill approximately the condition of background plasma particle flux equal to zero $[43,44]$, which is realistic for present tokamaks as well as for ITER in the usual condition of small particle source in the plasma core. The specific choice of the wave number, namely $k_{\theta} \rho_{i}=0.18$, at which the runs presented in this work are made, is motivated by the fact that this value is in the usual range where the nonlinear ion temperature gradient transport is maximum (e.g. Ref. [45]). Moreover analogous values, namely smaller than those at which the maximum linear growth rate is located, have been found to provide a good agreement between fully nonlinear simulations and quasi-linear calculations of fast particle transport similar to those presented here [4]. Of course, a quasi-linear treatment does not allow us to take into account the effects of nonlinear interactions between the fast particle motion and the background turbulence. The results of [4] suggest that, at least in plasma conditions such as those considered in the present work, these effects are small.

In Fig. 1 we show a $\alpha$ particle concentration scan. In these calculations, performed with the GS2 code with a slowing down equilibrium function for the $\alpha$ particles, the bulk background temperature has been taken to be a realistic ITER value, namely $T_{e}=T_{i}=10$ $\mathrm{keV}$. The $\alpha$ particle species has been considered with the same other physical dimensionless parameters as the plasma background. We observe that up to the maximum concentrations foreseen in the ITER standard scenario, namely $Z_{\alpha} n_{\alpha} / n_{e}=0.016$ [27], the growth rate of the mode is rather insensitive to the variation of the $\alpha$ charge concentration. A charge 
concentration of $2 \%$ modifies the growth rate of the mode by less than $4 \%$, while a concentration of $20 \%$ reduces the growth rate by more than $25 \%$. On one side, these results show that in the ITER standard scenario the fast particle concentration is too small to provide an effective mechanism for turbulence stabilization as instead observed for instance in AUG in the formation of internal transport barriers at low density with neutral beam injection heating [46]. On the other side, these results suggest that, particularly at midradius, where charge concentrations below $1 \%$ are expected, the behavior of the $\alpha$ particles is very close to that of the passive tracer limit. Since in this limit, the transport coefficients defined in the previous section become independent of the fast particle radial gradients, this limit not only is more suited for the kind of results we are looking for, but also remains realistic for the description of the transport of $\alpha$ particles in ITER. For this reason, all the calculations presented in the remainder of this paper are performed with a $\alpha$ particle charge concentration of $510^{-4}$, well inside the passive tracer limit (corresponding variation of the growth rate below 1/1000). We note that, however, the $\alpha$ particle species is treated as an active species by both the codes we have applied.

\section{DEPENDENCE ON THE ENERGY VARIABLE}

The dependence of the kernel function $G_{D}$, defined in Eq. (26), on the dimensionless energy variable $E / T_{e}$ is illustrated in Fig. 2. This has been computed with GS2 with background plasma parameters given by the reference case introduced in the previous section. We remind that, although the function $G_{D}\left(E / T_{e}\right)$ is independent of the specific choice of the equilibrium distribution function, it remains dependent on the physical parameters of the background plasma. As indicated by Eq. (27), the diffusion coefficient $D$ is given by the convolution of this function with the equilibrium distribution function. Therefore, the function $G_{D}$ can be interpreted as providing the actual dependence of the diffusivity on the energy variable in the velocity space. We find that the transport of the fast particles becomes negligible above the slowing down critical energy $E_{c} / T_{e}=33.05$. More precisely, 
as shown in the inset in logarithmic scale, particles with energies equal to 10 times the background temperature have a diffusivity reduced by almost one order of magnitude with respect to those which are in the thermal range. Therefore transport is produced only in the lowest energy range of the distribution, and the diffusivity decreases quickly with increasing energy. This can be understood as due to the combination of two effects. One is given by the decrease of the function $J_{0}^{2}$ with increasing energy (we remind that in Eq. (26) one $J_{0}$ is included in the solution $h_{D}$ ) . The second is given by the fact that only the slow particles are resonant in the denominator of the integrand of Eq. (21). Here the mode eigenfrequency $\omega$ is of the order of the drift frequency of the background thermal particles, and therefore it is only in the proximity of the corresponding thermal energy range that the phase shift between the density fluctuations of the fast particles and the electrostatic potential is significantly non-zero.

It is useful to derive a simple fitting formula of the function $G_{D}$ in terms of the dimensionless variable $E / T_{e}$. This reads,

$$
\begin{gathered}
G_{D}\left(E / T_{e}\right)=1.25 \text { for } E / T_{e} \leq 2.7 \\
G_{D}\left(E / T_{e}\right)=\exp \left[-8.1410^{-5}\left(E / T_{e}\right)^{4}+3.7710^{-3}\left(E / T_{e}\right)^{3}-\right. \\
\left.0.0553\left(E / T_{e}\right)^{2}+0.036\left(E / T_{e}\right)+0.45\right] \text { for } E / T_{e}>2.7,
\end{gathered}
$$

and it is shown in Fig. 2 by a dashed curve.

In Sec. VIII, we shall show that the gyrokinetic results presented in the following sections for the diffusion and convective coefficients, as computed directly by GS2 and GKW, can be also obtained, with good accuracy, by performing simple integrals of this fitting formula with the chosen distribution function, as indicated by the analytical derivation of the particle flux presented in Sec. III. 


\section{DEPENDENCE ON THE BACKGROUND TEMPERATURE OF THE DIFFUSION COEFFICIENT}

With a charge concentration of fast particles equal to $510^{-4}$, we have computed the diffusion coefficient $D_{\alpha}$ of the $\alpha$ particles as a function of the background electron temperature, or of the ratio $E_{\alpha} / T_{e}$, which is the same for these electrostatic collisionless calculations, since only the energy ratio matters in this case. In Fig. 3 , the coefficient $D_{\alpha}$ is plotted as a function of the background electron temperature. The coefficient has been normalized to the diffusion coefficient $D_{\text {Max }}\left(T_{\alpha}=T_{e}\right)$, namely the diffusion coefficient the $\alpha$ particles have in the case they are a thermal species at the same temperature of the bulk and described by a Maxwellian distribution. Such a diffusion coefficient used for normalization has a direct physical interpretation, namely it is the diffusion coefficient of He ash $D_{\mathrm{He}}$.

First of all, Fig. 3 shows the excellent agreement obtained between the two gyrokinetic codes, with differences below $5 \%$. The curve obtained with a slowing down distribution function is compared to the one obtained with an equivalent Maxwellian distribution. Small but non-negligible differences are obtained. In particular we note that, differently from the Maxwellian case, which shows a constant asymptotic behavior, already observed and explained analytically in [4], the curve obtained with the slowing down distribution function provides values of the $\alpha$ particle diffusion coefficient which are below those provided by an equivalent Maxwellian at large background electron temperatures, or correspondingly at low ratios $E_{\alpha} / T_{e}$, while it becomes larger at smaller background electron temperatures. Interestingly, the region at which the two curves cross is in between $10 \mathrm{keV}$ to $20 \mathrm{keV}$, which makes that the description in terms of an equivalent Maxwellian is applicable in the range of temperatures predicted for ITER. We observe as well that in the ITER range of background plasma temperatures, $D_{\alpha} / D_{\text {Max }}\left(T_{\alpha}=T_{e}\right)<0.05$, namely the diffusivity of the $\alpha$ particles is at least 20 times smaller than the diffusivity those particles would have if they where thermalized at the same temperature of the bulk.

On the one hand, these theoretically predicted small values of the diffusivity of the 
fast particles with respect to the diffusivity of thermal particles are consistent with the observation in beam blip experiments on TFTR that the amount of radial spreading of beam ions during slowing down is fairly small, implying a fast ion diffusion coefficient which is an order of magnitude smaller than typical thermal transport coefficients [18]. On the other hand, our theoretical results might appear in stark contradiction with the results of some recent theoretical studies (e.g. Ref. $[1,2,4]$ ). We shall show in Sec. X that, at least specifically with respect to Ref. [4] in which a gyrokinetic model analogous to the present one was applied, this contradiction is only apparent, and originated by the different normalizations of the particle transport applied in the two works.

The differences between the curve of the diffusivity in the slowing down case and that of the diffusivity in the equivalent Maxwellian case can be explained easily in terms of the different weight that the two unperturbed distributions give to different energy ranges, along the idea indicated by the analytical derivation of Sec. III. To show this effect in more detail, we explore the dependence of the diffusion coefficients as a function of the energy variable in the velocity space. In Fig. 4(a-c), we plot the derivative of the diffusion coefficients versus the energy variable, namely the integrand in the right hand side of Eq. (27), for three different values of the background electron temperature. In Fig. 4(d-f) the corresponding equilibrium distribution functions, slowing down and equivalent Maxwellian, are plotted. We observe that $\alpha$ particles are transported only in the very slow energy tail of the distribution, consistently with the curve of $G_{D}$ presented in Fig. 2.

Fig. 4(d-f) shows that, due to the displacement of $E_{c} / E_{\alpha}$ as a function of $T_{e} / E_{\alpha}$, the number of fast particles in this slow energy range provided by the slowing down distribution changes relatively to the equivalent Maxwellian. In particular for $T_{e}<20 \mathrm{keV}$, the slowing down distribution has more particles in the slow energy range, where fast particles are transported by the background turbulence, with a consequent value of $D_{S l D}>D_{M a x}$. Around $T_{e}=20 \mathrm{keV}$ the two distribution functions are very close in the resonant energy range, as illustrated by Fig. $4(\mathrm{e})$, with consequent $D_{S l D} \simeq D_{M a x}$. Finally, for $T_{e}>20 \mathrm{keV}$, there are more particles in the low energy range in the equivalent Maxwellian distribution 
rather than in the slowing down, with consequent $D_{S l D}<D_{M a x}$. We see therefore that the differences with respect to the equivalent Maxwellian distribution are a consequence of the modification of the slowing down distribution function produced by the variation of $E_{c} / E_{\alpha}$ as a function of $T_{e}$, which implies that different numbers of $\alpha$ particles are present in the resonant energy range where fast particles are transported.

A simple algebraic expression fitting the code results which provides the dependence of $D_{\alpha} /\left[D_{\text {Max }}\left(T_{\alpha}=T_{e}\right)\right]$ as a function of the background plasma temperature reads

$$
\frac{D_{\alpha}}{D_{\operatorname{Max}}\left(T_{\alpha}=T_{e}\right)}=0.02+4.5\left(\frac{T_{e}}{E_{\alpha}}\right)+8\left(\frac{T_{e}}{E_{\alpha}}\right)^{2}+350\left(\frac{T_{e}}{E_{\alpha}}\right)^{3},
$$

which is applicable in the range $5 \mathrm{keV}<T_{e}<150 \mathrm{keV}$, namely $23<E_{\alpha} / T_{e}<700$, with an error below $3 \%$ with respect to the original numerical results.

We have verified that such a dependence on $T_{e} / E_{\alpha}$ of the normalized diffusivity, shown in Fig. 3 for $k_{\theta} \rho_{i}=0.18$ and described by the fit of Eq. (33), is insensitive of the value of $k_{\theta} \rho_{i}$ at which the linear calculations are performed, in the usual range of long wave length instabilities, namely $k_{\theta} \rho_{i}<1$.

\section{DEPENDENCE ON THE BACKGROUND TEMPERATURE OF THE CONVECTIVE TERMS}

In this section we investigate the dependence of the convective coefficients $C_{E \alpha}=$ $D_{E \alpha} / D_{\alpha}$ and $C_{p \alpha}=R V_{p \alpha} / D_{\alpha}$ on the background electron temperature $T_{e}$. The results of the GS2 calculations, as well as those of GKW, with both the equilibrium slowing down and the equivalent Maxwellian distribution functions, are shown in Fig. 5. The results of the two codes for the slowing down case are found in excellent agreement also on these terms.

For both these coefficients, non-negligible differences are found with the two choices of unperturbed distribution functions. As it will be explained in detail, these arise from the different expressions of the functions $\mathcal{H}(\hat{E})$ and $\mathcal{K}(\hat{E})$ derived from a slowing down or from a Maxwellian. 
In Fig. 5(a), the coefficient related to the convective contribution proportional to the logarithmic gradient of the critical energy, namely proportional to $R / L_{T e}$, is plotted. For comparison we have included two curves for the Maxwellian case, one (open symbols) computed with the usual Maxwellian expression for $\mathcal{K}_{M}$, namely $\hat{E}-3 / 2$, the second (full symbols) computed for the appropriate expression for the Maxwellian equivalent to the corresponding slowing down function, namely $\mathcal{K}_{M}$ as provided by Eq. (16). We observe that the ratio $d \log T_{\alpha} / d \log T_{e}$ is around zero for $T_{e} \sim T_{\alpha}$ and increases up to around 0.2 for $T_{e}$ in the range of $10 \mathrm{keV}$ to $20 \mathrm{keV}$. The consequence of this is that the equivalent temperature profile of the $\alpha$ particles is rather flat, as already underlined in Ref. [4].

We observe that the curve with open symbols is positive (outward flux) for $T_{e} \simeq E_{\alpha}$, while it becomes negative with decreasing $T_{e}$ and approaches $-3 / 2$ for very small values of the electron temperature. This can be understood from Eqs. (23) and (24). In the limit $T_{e} \simeq E_{\alpha}, \hat{E}$ dominates over $-3 / 2$ in the integrand of Eq. (24), providing an overall positive term, while in the limit $T_{e}<<E_{\alpha}, \hat{E}$ can be neglected with respect to $-3 / 2$ in the expression of $\mathcal{K}_{M}(\hat{E})$, with the consequence that the ratio $D_{E k} / D_{k}$ reaches the asymptotic value of $-3 / 2$. The numerical results approach this analytical limit as it should be expected. The positive (outward) value of this term obtained for $T_{e} \simeq E_{\alpha}$ is in agreement with the direction of the thermodiffusion for impurities in the case of ITG instabilities $[47,48,39,49]$. This will be discussed more in detail at the end of Sec. IX, where a comparison with the He ash transport is made.

Once the curve in open symbols is multiplied by the appropriate ratio $d \log T_{\alpha} / d \log T_{e}$, we observe that the inward contribution provided by the equivalent Maxwellian (full squares) becomes smaller than that obtained with the slowing down distribution function (full circles). However, in both cases, the thermal convective contribution is directed inwards. This contribution, related to the temperature gradient, is the same that was observed in Ref. [4] to provide a He pinch. In the case a slowing down distribution is considered at the place of an equivalent Maxwellian, such an inward particle flux contribution is even larger.

In order to better understand the behavior of the result obtained with a slowing down 
equilibrium distribution function, in Fig. 6 we plot the analytic function $\mathcal{K}_{S}(\hat{E})$ provided by Eq. (12) as a function of $T_{e}$ for different values of $\hat{E}$. The reason of such an unusual way of plotting this function will appear clear below. In the limit of $T_{e} \sim E_{\alpha}$, $\mathcal{K}_{S}$ is equal to zero, since the two terms of opposite sign in the expression for $\mathcal{K}_{S}(\hat{E})$ balance in that limit. This is reflected by the fact that in the description provided by an equivalent Maxwellian, $d \log T_{\alpha} / d \log T_{e}$ is close to zero in the same limit. With decreasing $T_{e}, \mathcal{K}_{S}(\hat{E})$ provides an increasingly larger negative contribution for low values of $\hat{E}$, namely the second term dominates over the first in the right hand side of Eq. (12). For $\hat{E}=0, \mathcal{K}_{S}$ takes the values which are approached by the numerical results in the limit of $T_{e}<<E_{\alpha}$. The general consequence of this behavior is that the actual full numerical curve for $C_{E \alpha}$ is not significantly different from the curve $\mathcal{K}_{S}(\hat{E}=0)$. The latter can be used as analytical expression for this term in a simplified transport model for the $\alpha$ particles to be applied in transport simulations, as it will proposed in Sec. IX.

Even larger differences between the slowing down case and the equivalent Maxwellian case are found for the pure convection coefficient $C_{p \alpha}$. We observe however that in the range of temperatures $10 \mathrm{keV}<T_{e}<20 \mathrm{keV}$, the two curves are close and both very close to zero. On the contrary, for values of $E_{\alpha} \sim T_{e}$, the Maxwellian case provides a strong inward particle flux, while the slowing down distribution case has a flux close to zero. All these differences can be understood recalling Eqs. (23) and (25), and the different definitions of $\mathcal{H}_{S}$ and $\mathcal{H}_{M}$ in Eqs. (13) and (15) respectively. To this purpose, in Fig. 7 we have plotted $\mathcal{H}_{S}$ as a function of $\hat{E}$ for different values of $T_{e}$. We recall that in the Maxwellian case, this function is a constant equal to 1 . The behavior of $\mathcal{H}_{S}$ presented in Fig. 7 can be more easily understood by recasting the expression for $\mathcal{H}_{S}$ in the form

$$
\mathcal{H}_{S}(\hat{E})=\frac{3}{2} \frac{E_{\alpha}}{E_{c}} \frac{\left(E / E_{c}\right)^{0.5}}{\left(E / E_{c}\right)^{1.5}+1} .
$$

From this expression it is straightforward to observe that this function has maximum value $(0.5)^{1 / 3}\left(E / E_{c}\right)$ at $\left(E / E_{c}\right)^{1.5}=0.5$. Since $\mathcal{H}_{S}(\hat{E})$ is proportional to $E_{\alpha} / E_{c}$, we observe that it implies that $V_{p \alpha}$ has given by Eq. (25) has the same dependence with decreasing $T_{e} / E_{\alpha}$ 
of $D_{\alpha}$ for $T_{e}<<E_{\alpha}$, with consequent asymptotic constant behavior of $C_{p \alpha}$ in that limit, as found in the numerical results. Such a behavior is different from that obtained in the equivalent Maxwellian case, for which $\mathcal{H}_{M}(\hat{E})=1$ and therefore $V_{p \alpha}$ has an additional $1 / E_{\alpha}$ factor in front as compared to $D_{\alpha}$, which explains why in the equivalent Maxwellian case the asymptotic value of $C_{p \alpha}$ for $T_{e}<<E_{\alpha}$ is zero. In the range of temperatures $10 \mathrm{keV}<T_{e}<$ $20 \mathrm{keV}$, this pure convective contribution is negligible, by at least one order of magnitude, with respect to that provided by $C_{E \alpha} R / L_{T e}$. The effect of this pinch term can therefore be neglected in transport simulations of $\alpha$ particles.

\section{CALCULATION OF THE TRANSPORT COEFFICIENTS ADOPTING A FITTING FORMULA FOR THE ENERGY DEPENDENCE OF THE DIFFUSIVITY KERNEL FUNCTION}

In the present section, we provide an example of a general procedure that we propose with the purpose of computing transport coefficients for fast ions with any unperturbed distribution function. We utilize the formula introduced in Eq. (32), which fits the dependence of the kernel function $G_{D}$ on the energy variable in the velocity space, and by direct integration on the energy variable along the relations provided by Eqs. (27) and (28), we compute both the coefficients $D_{\alpha}$ and $C_{E}=D_{E} / D_{\alpha}$. We compare the results from these calculations, performed externally of the gyrokinetic codes, with the results of the codes, presented in the previous sections in Figs. 3 and 5. The comparison is shown in Fig. 8 and is performed over different values of the background temperature $T_{e}$, assuming both a slowing down and a Maxwellian distribution functions. We note that these have significantly different shapes, as shown, for instance, in Fig. $4(\mathrm{~d}-\mathrm{f})$ with increasing $T_{e}$. The calculations applying the formula provide diffusion and thermal convection coefficients which are in good agreement (within $10 \%$ ) with the original GS2 and GKW results, over a wide range of temperatures, and with both the slowing down and the Maxwellian distribution functions.

By this example, we demonstrate that such a procedure, which utilizes a fitting formula 
for the kernel function $G_{D}\left(E / T_{e}\right)$ to compute the transport coefficients, can be applied for general purposes, with any equilibrium distribution function. In particular, the method described in the present section is certainly of interest in the framework of gyrokinetic calculations of the transport of beam ions or radiofrequency $(\mathrm{RF})$ accelerated ions. We have to remind the caveat that the present derivation is performed exclusively for equilibrium distribution functions which are isotropic in the velocity pitch angle, namely which depend only on the energy variable. This condition is usually not fulfilled by the distribution functions of beam ions and even less by those of RF accelerated ions. However, we argue that the dependence of the equilibrium distribution function on the pitch angle is not likely to affect strongly the dependence on energy of the kernel function $G_{D}$. The main reason for this is that, differently from electrons, for which are usually exclusively the trapped particles to be transported, the ion particle transport is usually not a strong function of the velocity pitch angle. Then, in the case that the dependences on energy and on the velocity pitch angle can be expressed in separate factors in the unperturbed distribution function, a derivation analogous to that presented in Sec. III shows that the presence of a dependence on the pitch angle in the distribution function does not modify the energy dependence of the kernel function $G_{D}$, except for an overall factor in front which, for the reasons expressed above, is of the order unity, and which, in any case, can be estimated separately. On the other hand, it is worth mentioning that the presence of a dependence on the pitch angle in the equilibrium distribution function can introduce additional convective contributions in the total expression of the particle flux. Nevertheless, these arguments suggest that, for first order estimates, the function $G_{D}$, computed assuming an isotropic equilibrium distribution function as in the present work, can be used also for calculations of the diffusion coefficients with non-isotropic equilibrium distribution functions, like those of beam and RF accelerated ions. Moreover, a fitting formula of the kernel function $G_{D}$, like Eq. (32), can be also included in Fokker-Planck or Monte Carlo codes to describe the additional effect of the transport produced by the background turbulence on the slowing down process. 
In conclusion, the method described in the present section, making use of a fitting formula for the kernel function $G_{D}$, is proposed as a fast and simple procedure for very general purposes of gyrokinetic calculations of fast ion transport, in the experimentally applicable limit of small concentration (passive tracer). It is rigorous when applied with equilibrium distribution functions which depend only on the energy variable, while it provides only a first order estimate in the case of equilibrium distribution functions which depend also on the velocity pitch angle. Of course, the function $G_{D}$ depends on the background plasma parameters, and has to be recomputed when these change significantly. However, such a method can be applied without introducing any modification in a gyrokinetic code. Otherwise, the obtention of the exact solution directly within a gyrokinetic code implies the need of implementing a different version of the gyrokinetic code for each specific choice of the equilibrium distribution function.

\section{TRANSPORT MODEL FOR $\alpha$ PARTICLES}

The numerical results obtained with the codes GS2 and GKW and presented in the previous sections can be summarized in the form of a transport model for $\alpha$ particle transport. Here we gather together the formula for the diffusivity as a function of $E_{\alpha} / T_{e}$ in Eq. (33), and the analytical expression for the function $\mathcal{K}_{S}(\hat{E})$, evaluated at $\hat{E}=0$, which provides the appropriate description of the numerical results of the thermal convection coefficient $C_{E \alpha}$, as discussed in Sec. VII. Furthermore, we exclude the negligible contribution from $C_{P \alpha}$,

A simple algebraic expression which fits the numerical results in the range $23<E_{\alpha} / T_{e}<$ 700 , and provides the $\alpha$ particle flux as a function of the background electron temperature profile, for plasma conditions not far from those predicted for the ITER standard scenario, reads,

$$
\frac{R \Gamma_{\alpha}}{n_{\alpha}}=D_{\mathrm{He}}\left[0.02+4.5\left(\frac{T_{e}}{E_{\alpha}}\right)+8\left(\frac{T_{e}}{E_{\alpha}}\right)^{2}+350\left(\frac{T_{e}}{E_{\alpha}}\right)^{3}\right]\left(\frac{R}{L_{n \alpha}}+\mathcal{K}_{S}(0) \frac{R}{L_{T e}}\right),
$$


where $\mathcal{K}_{S}(0)$ is defined in Eq. (12) and is reported here below, with $E_{c}$ being the slowing down critical energy,

$$
\mathcal{K}_{S}(0)=\frac{3}{2}\left\{\frac{1}{\log \left[\left(E_{\alpha} / E_{c}\right)^{1.5}+1\right]\left[1+\left(E_{c} / E_{\alpha}\right)^{1.5}\right]}-1\right\},
$$

where we remind that $E_{c} / E_{\alpha}=33.05 T_{e} / E_{\alpha}$.

The above expression for the $\alpha$ particle flux is proposed for applications in transport modelling. The coefficient $D_{\mathrm{He}}=D_{\text {Max }}\left(T_{\alpha}=T_{e}\right)$ is the diffusivity of a He particle at the same temperature as the background electron temperature, namely the diffusivity of He ash, and can be computed consistently by the transport model applied in the simulation. We recall that the above formula is proposed for the description of fast $\alpha$ particles, and it is not applicable for the description of He ash, for which the treatment as a normal impurity, e.g. at the same temperature of the background plasma, is certainly more appropriate. It is interesting to compare the transport model for $\alpha$ transport introduced above with a transport model for He ash, derived in an analogous way, with the background plasma in the specific conditions of our reference case. We describe He ash as an impurity species at the same temperature as the background plasma with a Maxwellian distribution function. Therefore $D_{\mathrm{He}}=D_{\text {Max }}\left(T_{\alpha}=T_{e}\right)$. We note that the condition $T_{\alpha}=T_{e}$ is fulfilled at the electron temperature $T_{e}=1401 \mathrm{keV}$, therefore the values of $C_{E \mathrm{He}}$ and $C_{p \mathrm{He}}$ for the $\mathrm{He}$ ash can be obtained from the curves plotted in Fig. 5 at this value of the electron temperature (dash-dotted vertical lines). We note as well that for He ash the $C_{E \mathrm{He}}$ coefficient has to be considered as a thermodiffusion coefficient for a Maxwellian distribution, and therefore taken from the dashed curve with open symbols in Fig. 5(a). We find $C_{E H e}=0.03$ and $C_{p \mathrm{He}}=-2.2$, which leads to the following expression for the He ash particle flux,

$$
\frac{R \Gamma_{\mathrm{He}}}{n_{\mathrm{He}}}=D_{\mathrm{He}}\left(\frac{R}{L_{n \mathrm{He}}}+0.03 \frac{R}{L_{T \mathrm{He}}}-2.2\right) .
$$

The thermodiffusive term for He ash $\left(C_{E \mathrm{He}} R / L_{T \mathrm{He}}=0.174\right)$ is positive (outward) and small for our reference case, while the pure convection term (sometimes called "curvature pinch") is large and directed inward. These results are consistent with the well known directions 
of thermodiffusion and pure convection of impurities in the presence of ITG turbulence $[47,48,39,49]$. For comparison, we remind that for the fast $\alpha$ particles, we find that it is the thermal convection to be inward and large while the pure convection is still inward, but negligible $\left(C_{E \alpha} R / L_{T e}=-5.25\right.$ and $C_{p \alpha}=-0.137$ at $\left.T_{e}=15 \mathrm{keV}\right)$.

\section{COMPARISON WITH PREVIOUS RESULTS}

As we mentioned in the introduction, fast particle transport has been already the object of several studies in the past. The results reported in the literature however can differ even qualitatively, and contradictory statements can be found about whether the transport of fast particles is significantly smaller or as large as or even larger than the transport of the background turbulent plasma. Original works (e.g. [8]) concluded that, on the basis of simple orbit average arguments, the transport of energetic $\alpha$ particles produced by the background turbulence corresponds to diffusivities two orders of magnitude smaller than that of the background thermal plasma. Our numerical results give a fully quantitative measurement, and are found in agreement, within a reasonable factor 5, with that simple estimate. In the same period, other works [7,9], comparing the total quasi-linear particle fluxes of the fast particles with those of the background plasma, found contradictory situations, in which the fast particle fluxes were small [7], as well as situations in which the fast particles fluxes were comparable to those of the background plasma [9]. It was then concluded that the fast particle transport could be large, depending on the conditions of the background plasma [9]. Later, numerical simulations of electrostatic turbulence [10,11], using the HasegawaMima model [50], concluded that the diffusion rate of energetic test particles was strongly reduced by the large Larmor radius averaging effect. Recently, studies applying the so-called decorrelation trajectory method $[1,2]$, found the surprising and opposite result that in conditions of relatively strong turbulence, the diffusivity of particles can increase with increasing gyroradius of the particles, by up to several orders of magnitude. More recently, a more complete treatment by a similar approach [3] has shown that those results were not correct, 
and results consistent with those of Ref. [11] were found in the same limits. In the same period, within a fully consistent gyrokinetic treatment [4], using the widely benchmarked gyrokinetic code GYRO [51], it was found that the turbulent $\alpha$ particle transport at high temperatures "remains stronger than the deuterium transport". These gyrokinetic results were presented as being in qualitative agreement with those of Ref. [2], and to some extent in contradiction with the orbit average arguments of the above mentioned previous works $[4]$.

Since in the present work we are applying a gyrokinetic model, as well as gyrokinetic codes, completely analogous to that used in Ref. [4], an agreement between our results and those of Ref. [4] has to be expected. However, the results presented in the previous Sections, and in particular those of Sec. V and Sec. VI, providing very small values of $D_{\alpha}$ as compared to the corresponding thermal diffusivity, in the range of temperatures $10 \mathrm{keV}<T_{e}<20$ $\mathrm{keV}$, could be considered opposite to those presented in Ref. [4]. We felt therefore bound to include this section, in which the specific problem of the comparison of our results with previous works in the literature is considered. We do hope that this section will help, at least partly, to reconcile the otherwise rather controversial situation of the literature described above.

In the following, we show that our results, while they agree qualitatively with the past simple orbit average estimates [8], as already pointed out above, at the same time they are not in disagreement with the recent gyrokinetic results [4].

In particular, we demonstrate that the disagreement of our results with those of Ref. [4] is only apparent, and caused by the different normalizations applied in the two works, and is not due to a major disagreement between the codes used in the present work, GS2 and GKW, and the GYRO code used in Ref. [4].

To this purpose, in Fig. 9(a) we plot our results for the same reference case as the one studied in Ref. [4], the General Atomics (GA) (or Waltz) standard case. Also in our results, when the same normalization as in Ref. [4] is adopted, a flux of $\alpha$ particles much larger than the deuterium particle flux is found when the $\alpha$ particles have comparable temperature to 
the deuterium. In the range of expected temperatures $10 \mathrm{keV}<T_{e}<20 \mathrm{keV}$, the flux of $\alpha$ particles remains larger than the reference deuterium flux, in the equivalent Maxwellian case. We note that the $\alpha$ particle flux of the slowing down case is slightly lower, mainly due to the larger inward contribution of the thermal convection coefficient $C_{E \alpha}$ in the case of a slowing down unperturbed distribution, as shown in Fig. 5(a), in Sec. VII. In Fig. 9 (b), we plot the same results in terms of the normalization applied in the present work. The $\alpha$ diffusion coefficient is normalized to the diffusion coefficient of He ash, namely the diffusion coefficient that $\alpha$ particles have if they are thermal at the same temperature of the background plasma, with a Maxwellian distribution. With this normalization, in the range $10 \mathrm{keV}<T_{e}<20 \mathrm{keV}$, very small values of the normalized diffusivity are found, below 0.07 .

For a more detailed and relevant comparison, we compute the ratio of the $\alpha$ particle flux per particle to the He ash flux per particle, namely $\left(\Gamma_{\alpha} / n_{\alpha}\right) /\left(\Gamma_{\mathrm{He}} / n_{\mathrm{He}}\right)$, and we compare this ratio with the result presented in Ref. [4], adopting, of course, the same input parameters (see Table IV of Ref. [4]), and treating $\alpha$ particles by an equivalent Maxwellian as made in that Reference. For $\alpha$ particles, at $T_{e}=15 \mathrm{keV}$, namely $T_{\alpha}=896 \mathrm{keV}$, we find $\left(R / L_{n \alpha}=15\right.$, $\left.R / L_{T \alpha}=1.5\right)$,

$$
\frac{R \Gamma_{\alpha}}{n_{\alpha}}=0.053 \times D_{\mathrm{He}} \times(15-1.34 \times 1.5-0.20)=0.678 D_{\mathrm{He}} .
$$

For He ash, we find $\left(R / L_{n \mathrm{He}}=3, R / L_{T \mathrm{He}}=9\right)$,

$$
\frac{R \Gamma_{\mathrm{He}}}{n_{\mathrm{He}}}=D_{\mathrm{He}} \times(3+0.085 \times 9-2.71)=1.055 D_{\mathrm{He}} .
$$

The ratio $\left(\Gamma_{\alpha} / n_{\alpha}\right) /\left(\Gamma_{\mathrm{He}} / n_{\mathrm{He}}\right)=0.64$, is in good quantitative agreement (difference below $10 \%)$ with the value obtained in Ref. [4], and shows clearly that comparable fluxes per particle are obtained for $\alpha$ particles and He ash, as found already in Ref. [4], although the diffusivities are in the ratio 1/20 (we find a factor 0.053 as quoted above), consistently with the results of the present work.

The plots in Fig. 9 as well as the numerical comparison made above show that the disagreement is only apparent, and is originated by the different normalizations applied. We 
note in particular that in Fig. 9(a) we have been considering the ratio between two particle fluxes which not only are obtained from significantly different density and temperature gradients, but which are actually unrelated. As a demonstration of this, the Reader can figure out the case in which the normalization adopted in Fig. 9(a) is applied to a realistic reference case, namely a case like the one adopted in this work in the previous sections, in which for the background plasma $\Gamma_{D} \simeq \Gamma_{e} \simeq 0$. In this case, adopting the normalization proposed in Ref. [4], arbitrarily large values of the fluxes of the $\alpha$ particles would have been found with respect to the background deuterium flux, since the latter is close to zero.

Besides this, we note that the value of $D_{\alpha} /\left[D_{\operatorname{Max}}\left(T_{\alpha}=T_{e}\right)\right]$ obtained with the GA standard case at $T_{e}=10 \mathrm{keV}$ is around 0.05 , and therefore is larger than the value obtained with the reference case applied in the previous sections in this work, for which $D_{\alpha} /\left[D_{\text {Max }}\left(T_{\alpha}=T_{e}\right)\right]=0.034$. This difference can be understood from Eq. (21). The larger is the (complex) frequency of the unstable mode produced by the background plasma, namely, roughly speaking, the more the mode is unstable, the larger is the number of fast particles fulfilling the resonance condition and contributing to the fast particle transport. The ITG mode of the GA standard case, with $R / L_{T}=9$, is significantly more unstable than the ITG mode of the reference case applied in this work with $R / L_{T}=6$, which explains this difference. We note that such an effect is only partly included in our normalization for $D_{\alpha}$, and would require an additional investigation of the dependence of $D_{\alpha} /\left[D_{M a x}\left(T_{\alpha}=T_{e}\right)\right]$ as a function of the background plasma parameters, to be fully described. Such an investigation goes beyond the scope of the present work. We have however performed a set of gyrokinetic calculations with GS2 and verified that the curves shown in Figs. 2 and 3 are not strongly modified by limited variations of the background plasma parameters around our reference case. This allows us to propose the fits in Eqs. (32), (35) and (36) for rather general purposes of $\alpha$ transport modelling.

In conclusion, in this section we have shown that no real strong disagreement is present between the results of this work and those of Ref. [4]. In our opinion, this reconciles qualitatively the results of the latter Reference with those of previous works, in which a reduction 
of the diffusion rate was found with increasing energy of the particles. We believe that the normalization for the $\alpha$ particle transport adopted in the present work is physically more meaningful. Here the actual diffusivity (and not the flux or the effective diffusivity) of the fast $\alpha$ particles is compared to the diffusivity that the particles would have if they were thermal, at the same temperature as the background plasma, namely the diffusivity of He ash.

\section{CONCLUSIONS}

In this work, we have presented an investigation using a quasi-linear gyrokinetic model for the transport of $\alpha$ particles with a slowing down equilibrium distribution function, in small concentration. The explicit form of the gyrokinetic equation with such an unperturbed distribution function has been derived and the consequences of this on the expression of the quasi-linear particle flux have been identified analytically and compared to the equivalent Maxwellian case. It has been shown that the transport coefficients can be elegantly expressed as integrals of an energy dependent kernel function, independent of the specific choice of the equilibrium distribution, times the chosen equilibrium function, with the inclusion of related auxiliary energy dependent functions, which are needed for the calculation of the convective terms.

The new terms in the right hand side of the gyrokinetic equation provided by the slowing down equilibrium distribution have been implemented in two gyrokinetic codes. Diffusive and convective contributions to the total $\alpha$ particle flux have been computed numerically, and a good agreement between the two codes has been found, ensuring the correctness of the implementation. The dependences on the background plasma electron temperature, or on the ratio of the birth energy of the $\alpha$ particles to the background temperature, have been investigated. Small but non-negligible differences with respect to the equivalent Maxwellian case have been found in the dependence of the $\alpha$ diffusivity on the bulk plasma $T_{e}$. In the range of electron temperatures predicted for the ITER standard scenario, the assumption 
of an equivalent Maxwellian, as made in Ref. [4], provides a reasonable approximation, with diffusivities which differ by less than $40 \%$ as compared with those computed with a slowing down distribution. Larger differences have been found in the dependences of the convective coefficients. All these differences have been explained in detail as a direct consequence of the presence of different terms in the gyrokinetic equation introduced by the more realistic assumption of a slowing down distribution function for the energetic $\alpha$ particles, consistently with the results of the analytical derivation.

The main physical results of this work are that for electron temperatures $T_{e}<20 \mathrm{keV}$, the $\alpha$ particle diffusivity $D_{\alpha}$ is at least 20 times smaller than the diffusivity $\alpha$ particles would have if they were thermal at the same temperature of the background plasma, namely the diffusivity of He ash. Moreover, it has been shown that, for a given value of the electron temperature, the $\alpha$ particles are transported only in the very slow energy range of the distribution, and no fast particle transport occurs above the slowing down critical energy.

Despite $\alpha$ particle diffusivities are a factor 20 smaller than He ash diffusivity, $\alpha$ particles and He ash can have comparable fluxes per particle, mainly as a consequence of the different density gradients, the $\alpha$ particle density being significantly more peaked, as already pointed out in Ref. [4]. By a detailed comparison, our results have been shown to be in agreement with those presented in Ref. [4]. This reconciles the results of this Reference with those of previous works, e.g. $[8,10,11,3]$, which find a decrease of the fast particle diffusion rate with increasing energy.

Inspired by the analytical derivation of the $\alpha$ particle flux, in which a kernel function is introduced which captures the dependence on the energy variable, independently of the specific shape of the equilibrium distribution function, a general procedure has been outlined by which transport coefficients can be computed for whatever choice of the equilibrium distribution. This utilizes a fitting formula of the energy dependence of the kernel function for the fast particle diffusivity as a function of the energy variable in the velocity space. As a demonstration, a simple analytical formula fitting this dependence as a function of the dimensionless energy variable $E / T_{e}$ has been derived in Eq. (32). By simple integrations 
on the energy variable, the formula has been shown to provide diffusion coefficients in good agreement with the original code results over a wide range of variation of the background electron temperature, and with both the slowing down and the Maxwellian distribution functions. A similar procedure is proposed to be used for direct applications in FokkerPlanck and Monte Carlo codes, for instance in the calculation of the transport of beam and radiofrequency accelerated ions.

A simple analytical expression for the $\alpha$ particle flux as a function of the ratio $E_{\alpha} / T_{e}$ has been derived, which fits the numerical results. This provides a transport model for $\alpha$ particles, as a function of the background electron temperature profile, applicable in the range $23<E_{\alpha} / T_{e}<700$, with plasma conditions not far from those predicted for the ITER standard scenario. The transport model is reported in Eqs. $(35,36)$.

\section{ACKNOWLEDGMENTS}

The Authors are grateful to W. Dorland and M. Kotschenreuther for providing the gyrokinetic code GS2, and to W. Dorland and G. W. Hammett for the web documentation of the code. The Authors are also grateful to J. Candy, G. W. Hammett and R. E. Waltz for fruitful comments and constructive suggestions to improve and complete the manuscript, and to A. Bottino, E. Fable and T. Hauff for helpful discussions.

[1] M. Vlad and F. Spineanu, Plasma Phys. Controlled Fusion 47, 281 (2005).

[2] M. Vlad, F. Spineanu, S. I. Itoh, M. Yagi, and K. Itoh, Plasma Phys. Controlled Fusion 47, $1015(2005)$.

[3] T. Hauff and F. Jenko, Phys. Plasmas 13, 102309 (2006).

[4] C. Estrada-Mila, J. Candy and R. E. Waltz, Phys. Plasmas 13, 112303 (2006). 
[5] T. Dannert, T. Hauff, F. Jenko, and S. Günter, CP871, Theory of Fusion Plasmas: Joint Varenna-Lausanne International Workshop, edited by J. W. Connor,O. Sauter, and E. Sindoni, American Institute of Physics 978-0-7354-0376-5/06, 51 (2006).

[6] T. Hauff and F. Jenko, Phys. Plasmas 14, 092301 (2007).

[7] G. Rewoldt, Phys. Fluids 31, 3727 (1988).

[8] R. B. White and H. E. Mynick, Phys. Fluids B 1, 980 (1989).

[9] G. Rewoldt, Nucl. Fusion 31, 2333 (1991).

[10] G. Manfredi and R. O. Dendy, Phys. Rev. Lett. 76, 4360 (1996).

[11] G. Manfredi and R. O. Dendy, Phys. Plasmas 4, 628 (1997).

[12] M. Vlad, F. Spineanu, J.H. Misguich, and R. Balescu, Phys. Rev. E 58, 7359 (1998).

[13] S. V. Annibaldi, G. Manfredi, and R. O. Dendy, Phys. Plasmas 9, 791 (2002).

[14] Editors of 'Progress in the ITER Physics Basis', Nucl. Fusion 47, S1 (2007).

[15] S. Günter, G. Conway, S. daGraca, H.-U. Fahrbach, C. Forest, M. Garcia Muñoz, T. Hauff, J. Hobirk, V. Igochine, F. Jenko, K. Lackner, P. Lauber, P. McCarthy, M. Maraschek, P. Martin, E. Poli, K. Sassenberg, E. Strumberger, G. Tardini, E. Wolfrum, H. Zohm and ASDEX Upgrade Team, Nucl. Fusion 47, 920 (2007).

[16] J. D. Strachan, P. L. Colestock, S. L. Davis, et al., Nucl. Fusion 21, 67 (1981).

[17] P. C. Efthimion, M. Bitter, E. D. Fredrickson, et al., in Plasma Physics and Controlled Nuclear Fusion Research 1988 (IAEA, Vienna, 1989), Vol. 1, p. 307.

[18] W. W. Heidbrink, Cris W. Barnes, G. W. Hammett, et al., Phys. Fluids B 3, 3167 (1991).

[19] W. W. Heidbrink, G. J. Sadler, Nucl. Fusion 34, 535 (1994).

[20] A. Fasoli, C. Gormenzano, H. L. Berk, B. Breizman, S. Briguglio, D.S. Darrow, N. Gorelenkov, 
W. W. Heidbrink, A. Jaun, S. V. Konovalov, R. Nazikian, J.-M. Noterdaeme, S. Sharapov, K. Shinohara, D. Testa, K. Tobita, Y. Todo, G. Vlad and F. Zonca, Nucl. Fusion 47, S264 (2007).

[21] W. W. Heidbrink, Phys. Plasmas 15, 055501 (2008).

[22] H. E. Mynick and R. E. Duvall, Phys. Fluids B 1, 750 (1989).

[23] E. Strumberger, S. Günter, E. Schwarz, C. Tichmann and the ASDEX Upgrade Team, New J. Phys. 10, 023017 (2008).

[24] E. Poli, M. García-Muñoz, H.-U. Fahrbach, S. Günter and the ASDEX Upgrade Team, Observation and modeling of fast trapped ion losses due to Neoclassical Tearing Modes, to be published in Phys. Plasmas (March or April 2008).

[25] S. S. Abdullaev, A. Wingen, and K. H. Spatschek, Phys. Plasmas 13, 042509 (2006).

[26] P. Pommois, G. Zimbardo, and P. Veltri, Phys. Plasmas 14, 012311 (2007).

[27] ITER Physics Basis Editors, Nucl. Fusion 39, 2175 (1999).

[28] M. Kotschenreuther, G. Rewoldt, W. M. Tang, Comp. Phys. Comm. 88, 128 (1995).

[29] W. Dorland, F. Jenko, M. Kotschenreuther, B. N. Rogers, Phys. Rev. Lett. 85, 5579 (2000).

[30] A. G. Peeters, D. Strinzi, Phys. Plasmas 11, 3748 (2004).

[31] A. G. Peeters, C. Angioni and D. Strinzi, Phys. Rev. Lett. 98, 265003 (2007).

[32] J. G. Cordey, W. G. F. Core, Phys. Fluids 17, 1626 (1974).

[33] J. D. Gaffey, Jr., J. Plasma Phys. 16, 149 (1976).

[34] D.E. Post, J. Fusion Energy 1, 129 (1981).

[35] W. G. F. Core, Nucl. Fusion 33, 829 (1993).

[36] P. J. Catto, Plasma Phys. 20, 719 (1978). 
[37] P. J. Catto, W. M. Tang and D. E. Baldwin, Plasma Phys. 23, 639 (1981).

[38] J. W. Connor, R. J. Hastie and J. B. Taylor, Phys. Rev. Lett. 40, 396 (1978).

[39] C. Angioni and A. G. Peeters, Phys. Rev. Lett. 96, 095003 (2006).

[40] C. Angioni, R. Dux, E. Fable, A. G. Peeters, Plasma Phys. Controlled Fusion 49, 2027 (2007).

[41] R. E. Waltz, G. M. Staebler, W. Dorland, G. W. Hammett, M. Kotschenreuther,and J. A. Konings, Phys. Plasmas 4, 2482 (1997).

[42] G. V. Pereverzev, C. Angioni, A. G. Peeters, O. V. Zolotukhin, Nucl. Fusion 45, 221 (2005).

[43] C. Angioni, A. G. Peeters, F. Jenko, T. Dannert, Phys. Plasmas 12, 112310 (2005).

[44] C. Angioni, L. Carraro, T. Dannert, N. Dubuit, R. Dux, C. Fuchs, X. Garbet, L. Garzotti, C. Giroud, R. Guirlet, F. Jenko, O. J. W. F. Kardaun, L. Lauro-Taroni, P. Mantica, M. Maslov, V. Naulin, R. Neu, A. G. Peeters, G. Pereverzev, M. E. Puiatti, T. Pütterich, J. Stober, M. Valovic , M. Valisa, H. Weisen, and A. Zabolotsky, Phys. Plasmas 14, 055905 (2007).

[45] Z. Lin and T. S. Hahm, Phys. Plasmas 11, 1099 (2004).

[46] G. Tardini, J. Hobirk, V. G. Igochine, C. F. Maggi, P. Martin, D. McCune, A. G. Peeters, A. C. C. Sips, A. Stäbler, J. Stober and the ASDEX Upgrade Team, Nucl. Fusion 47, 280 (2007).

[47] M. Frojdh, Liljestrom M, H. Nordman, Nucl. Fusion 32, 419 (1992).

[48] X. Garbet, N. Dubuit, E. Asp, Y. Sarazin, C. Bourdelle, P. Ghendrih, G.T. Hoang, Phys. Plasmas 12, 082511 (2005).

[49] N. Dubuit, X. Garbet, T. Parisot, R. Guirlet, and C. Bourdelle, Phys. Plasmas 14, 042301 (2007).

[50] A. Hasegawa and K. Mima, Phys. Fluids 21, 87 (1978).

[51] J. Candy, R. E. Waltz, J. Comput. Phys. 186, 545 (2003). 
Table 1. Values of $T_{e}$ and corresponding values of $E_{\alpha} / T_{e}, T_{\alpha}$ and $T_{\alpha} / T_{e}$ used in the gyrokinetic numerical calculations with GS2.

\begin{tabular}{rrrr}
\hline \hline$T_{e}[\mathrm{keV}]$ & $E_{\alpha} / T_{e} T_{\alpha}[\mathrm{keV}]$ & $T_{\alpha} / T_{e}$ \\
\hline 5 & 700.0 & 692.1 & 138.4 \\
8 & 437.5 & 772.7 & 96.6 \\
10 & 350.0 & 814.6 & 81.5 \\
15 & 233.3 & 896.1 & 59.7 \\
20 & 175.0 & 957.3 & 47.9 \\
30 & 116.7 & 1045.5 & 34.8 \\
40 & 87.5 & 1107.1 & 27.7 \\
60 & 58.3 & 1188.0 & 19.8 \\
90 & 38.9 & 1256.9 & 14.0 \\
150 & 23.3 & 1321.1 & 8.8 \\
250 & 14.0 & 1361.0 & 5.4 \\
500 & 7.00 & 1387.9 & 2.8 \\
1000 & 3.50 & 1398.4 & 1.4 \\
2000 & 1.75 & 1402.2 & 0.7 \\
3500 & 1.00 & 1403.4 & 0.4 \\
\hline \hline
\end{tabular}

Table 1. 


\section{Figure Captions}

FIG. 1. (Color online) GS2 calculations of the growth rate as a function of the charge concentration of the $\alpha$ particles for the reference case described in the text. The dashed-dotted vertical line shows the concentration value adopted in all the other gyrokinetic calculations presented in this paper.

FIG. 2. (Color online) Kernel function $G_{D}$, defined in Eq. (26), as computed by GS2 for the reference case parameters introduced in Sec. IV, as a function of the dimensionless energy variable $E / T_{e}$ (solid line). The dashed curve shows the approximation with the fitting formula in Eq. (32). In the inset, the curve computed by GS2 is plotted in logarithmic scale.

FIG. 3. (Color online) GS2 and GKW calculations of the diffusivity of the $\alpha$ particles for the slowing down (circles, solid line) and the equivalent Maxwellian (squares, dashed line) cases as a function of the electron temperature of the background plasma normalized to the diffusivity the $\alpha$ particles have in case they are thermal, with $T_{\alpha}=T_{e}$ and a Maxwellian equilibrium distribution function. The dash-dotted vertical line shows the temperature $T_{e}=1401 \mathrm{keV}$ for which $T_{\alpha}=T_{e}$.

FIG. 4. (Color online) Dependence of the energy derivative of the diffusion coefficient (a-c) and of the equilibrium distribution function (d-f) as a function of the energy variable in the velocity space for both the slowing down (solid line) and the equivalent Maxwellian (dashed line) cases for three different values of the background electron temperature. The dashed vertical line shows the slowing down critical energy.

FIG. 5. (Color online) GS2 and GKW calculations of the the convective coefficients $C_{E \alpha}$ and $C_{p \alpha}$ as a function of the background plasma electron temperature for the slowing down (circles, solid line) and the equivalent Maxwellian (squares, dashed line) cases, as well as for the normal Maxwellian (open squares, dashed line). The dash-dotted vertical line shows the temperature $T_{e}=1401 \mathrm{keV}$ for which $T_{e}=T_{\alpha}$. 
FIG. 6. (Color online) Dependence of the function $\mathcal{K}_{S}(\hat{E})$ as a function of the background electron temperature for different values of the energy variable $E / E_{\alpha}$. Curves are labelled in the plot.

FIG. 7. (Color online) Function $\mathcal{H}_{S}(\hat{E})$ as a function of the energy variable $E / E_{\alpha}$ for different values of the background electron temperature. Curves are labelled in the plot.

FIG. 8. (Color online) Diffusivity $D_{\alpha}$ (a) and thermal convection coefficient $C_{E}$ (b) computed with GS2 (full symbols) and recomputed using the formula in Eq. (32) for the kernel function $G_{D}$, along the integrals defined in Eq. (27) and Eq. (28) respectively (open symbols).

FIG. 9. (Color online) Flux of the $\alpha$ particles normalized to the flux of the background deuterium (a) and $\alpha$ diffusivity normalized to the $\alpha$ thermal diffusivity, with a Maxwellian distribution, as a function of the background electron temperature for the GA standard case. 


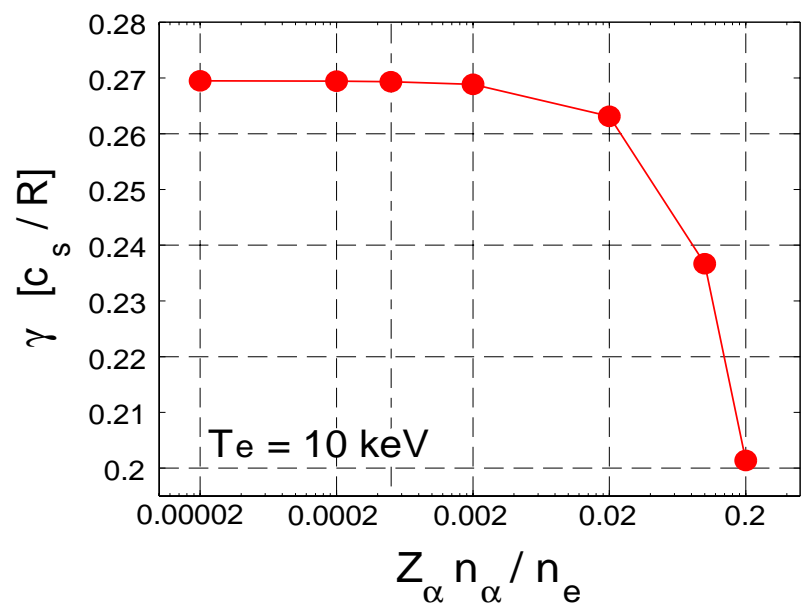

Figure 1

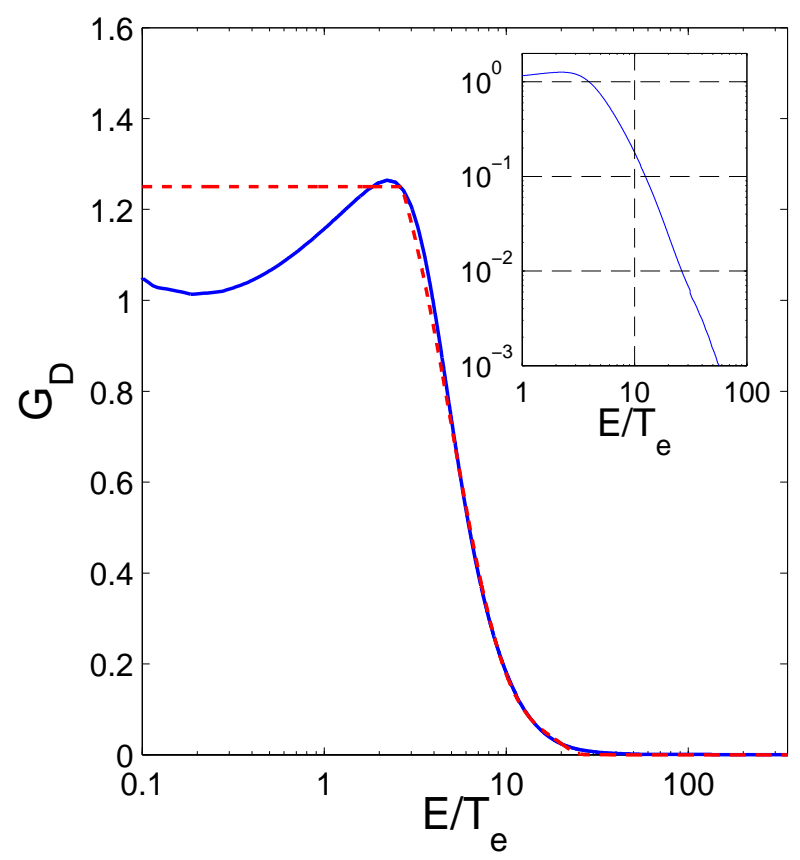

Figure 2 


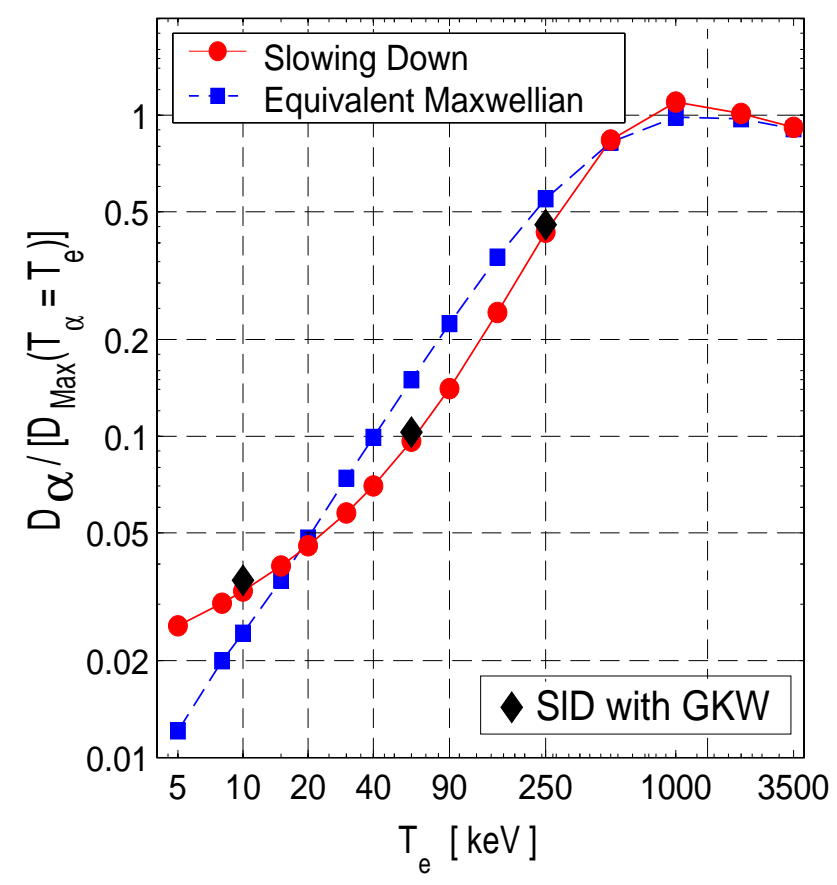

Figure 3 

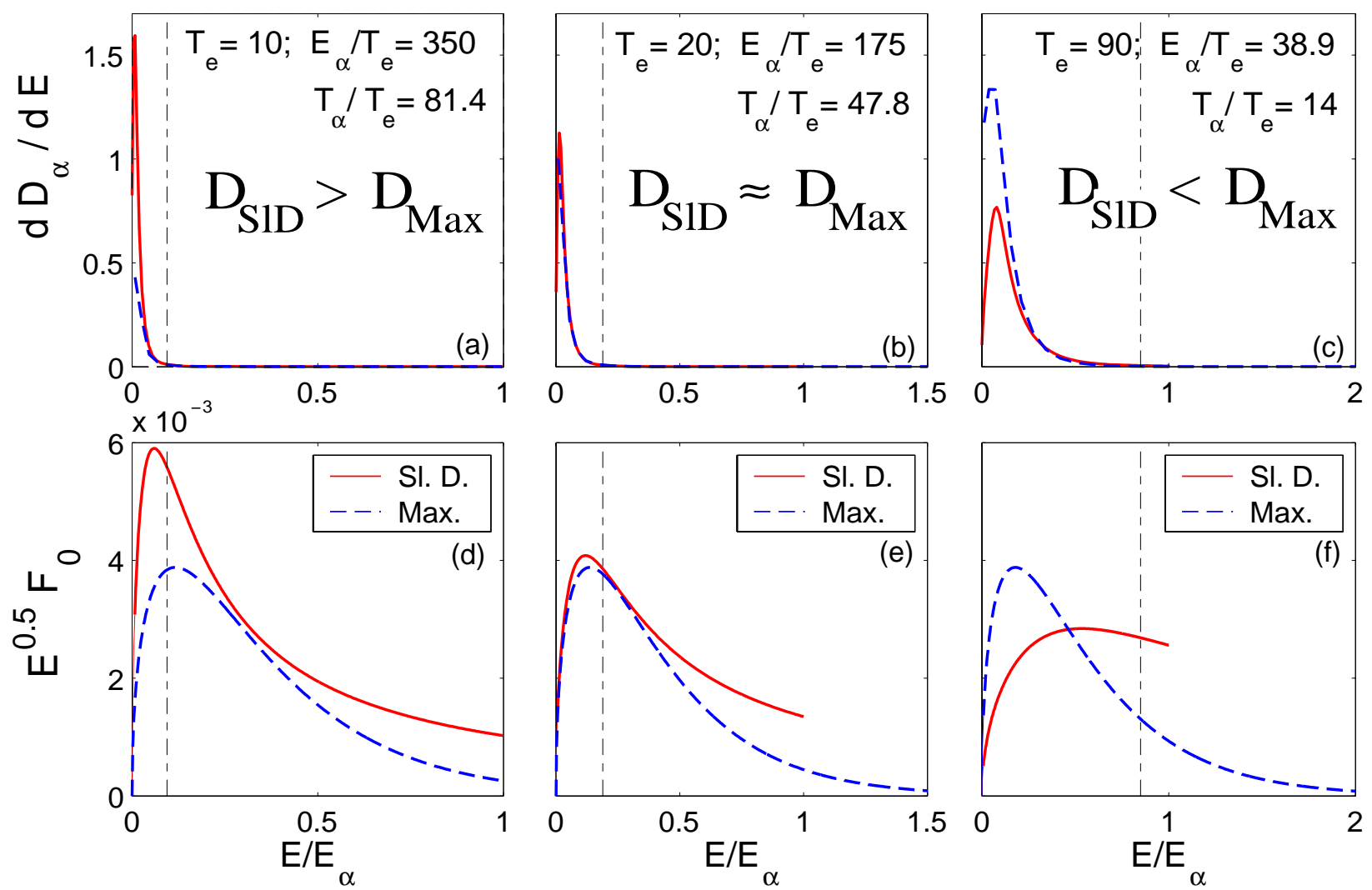

Figure 4 


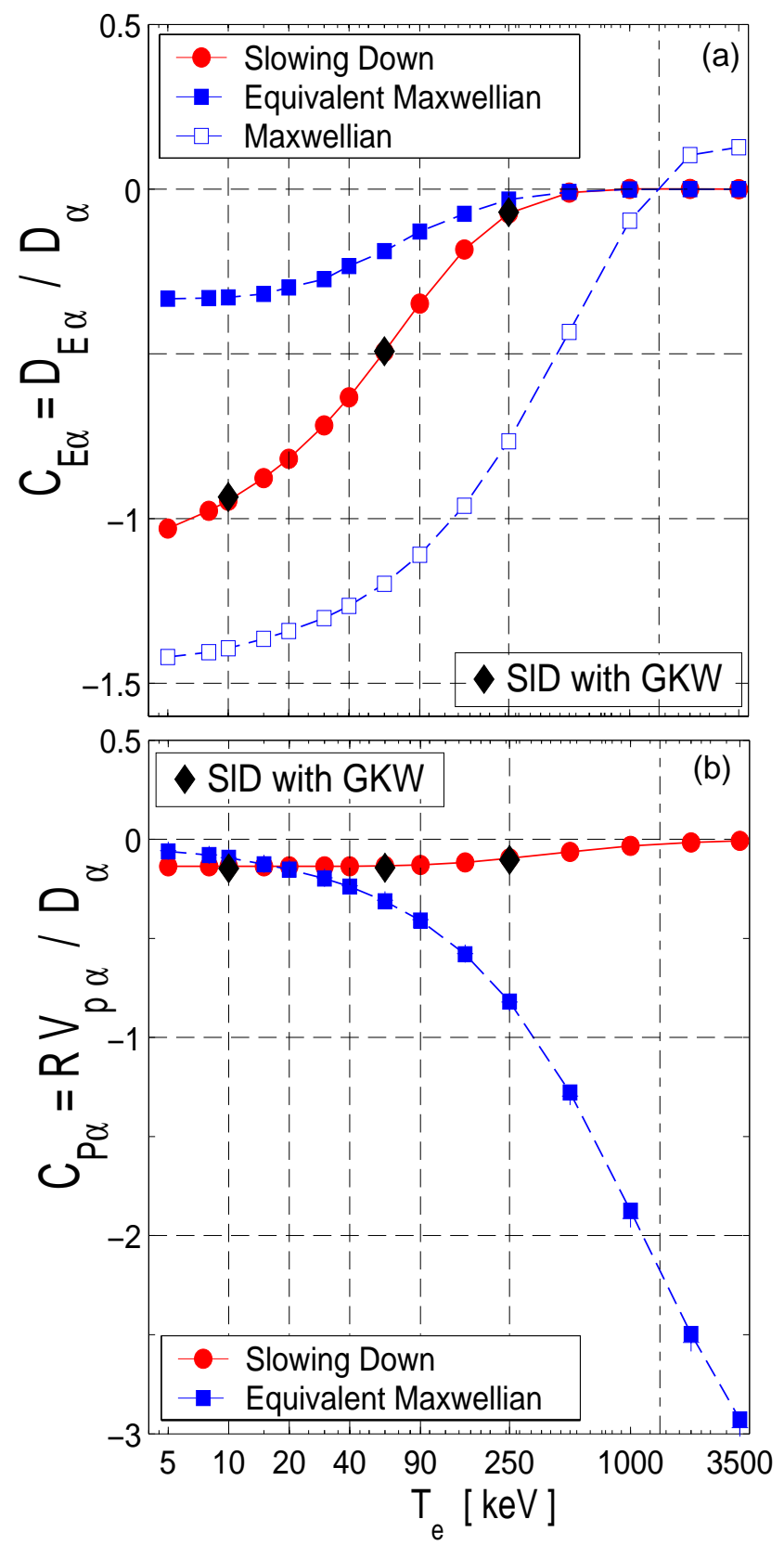

Figure 5 


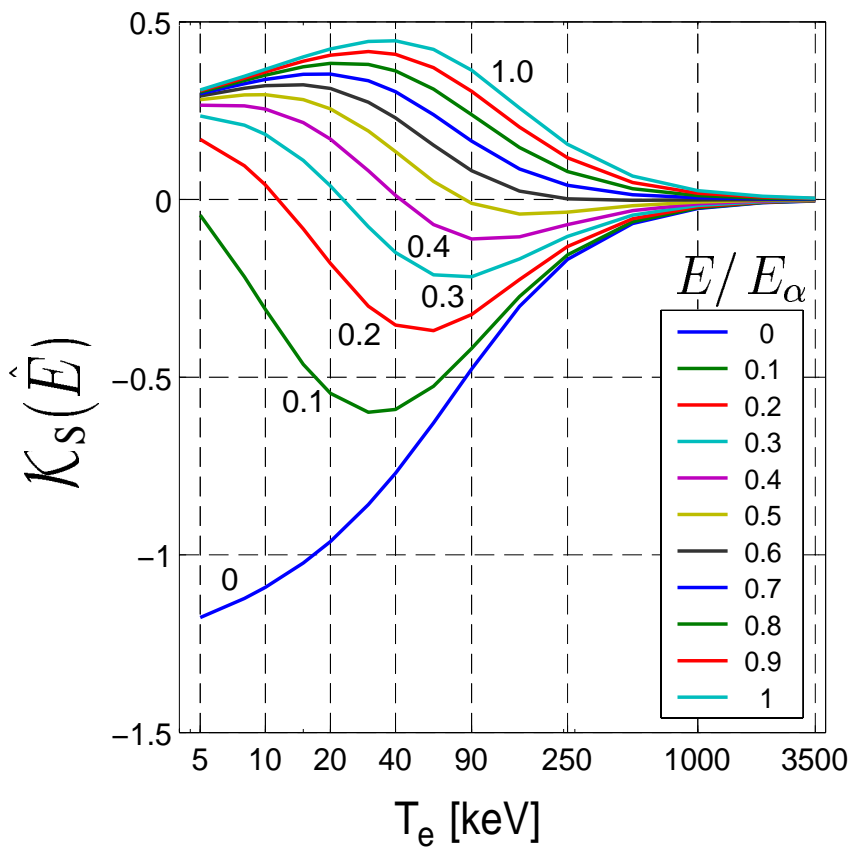

Figure 6

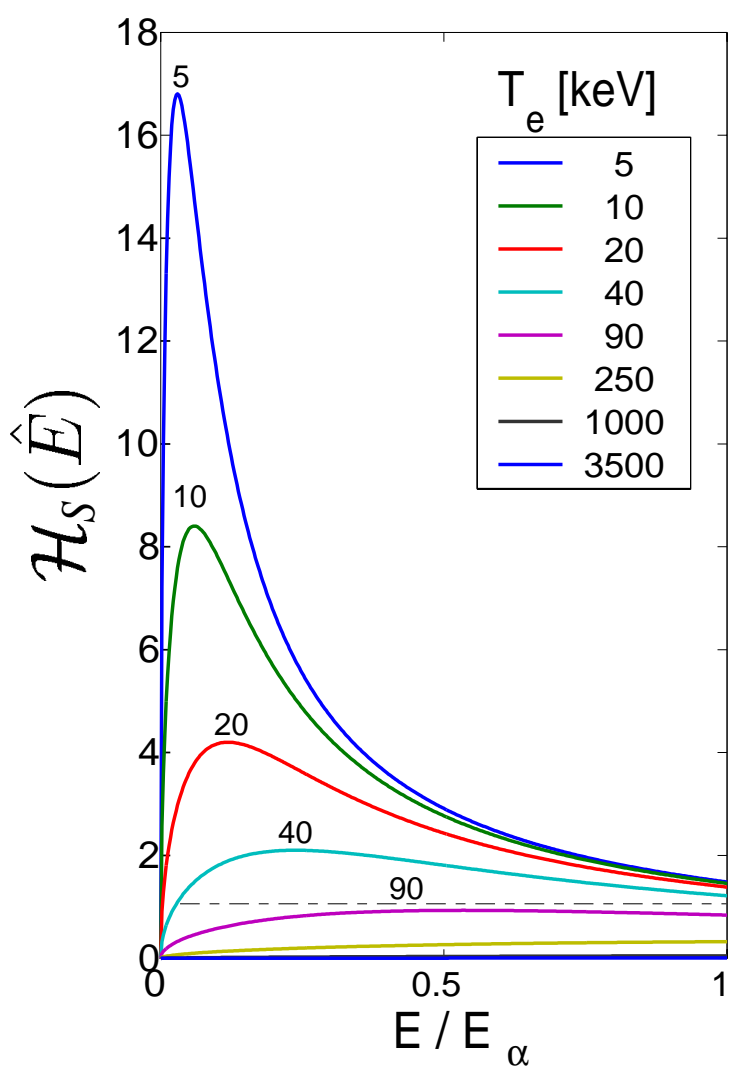

Figure 7 



Figure 8 


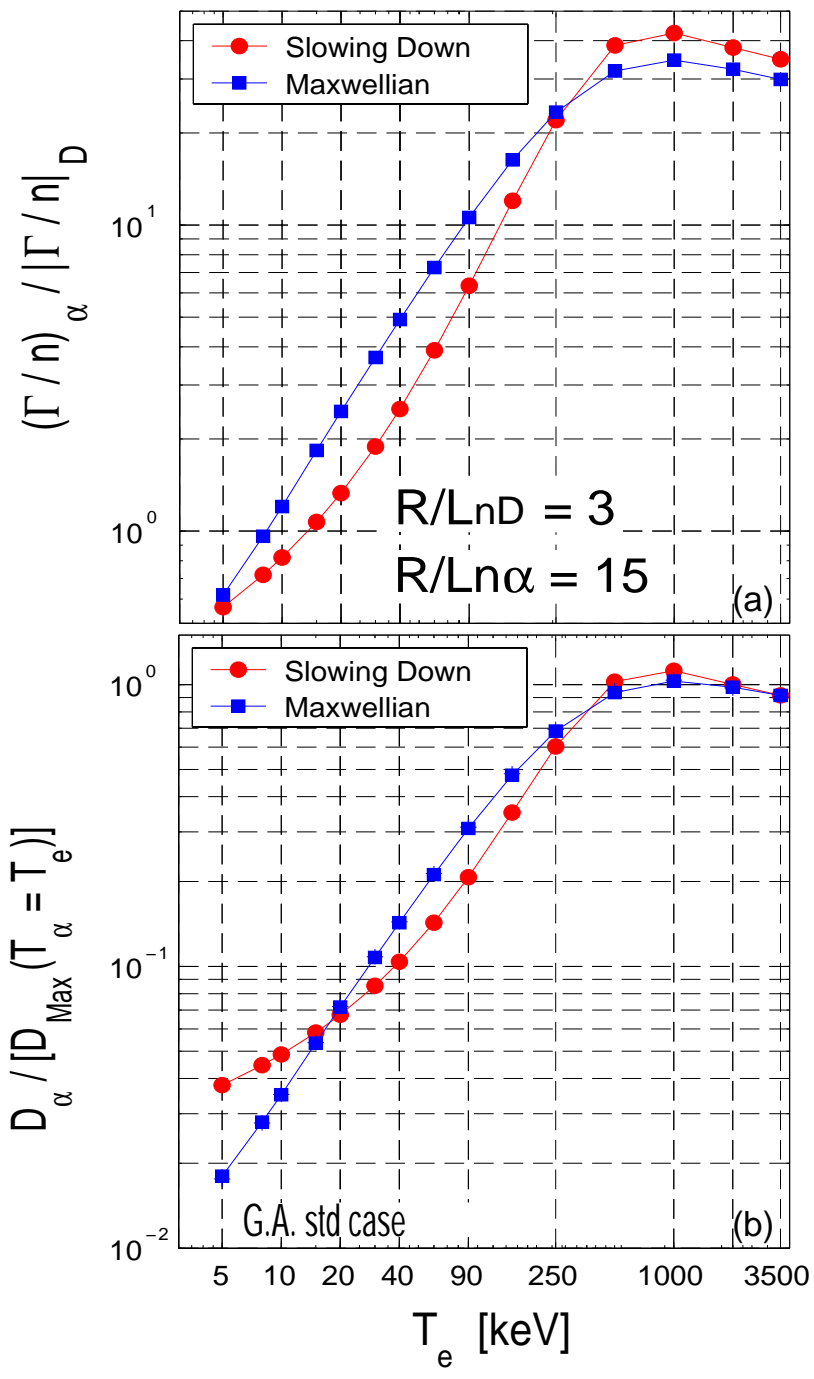

Figure 9 